\title{
Nature of the phase transition of the three-dimensional isotropic Heisenberg spin glass
}

\author{
Koji Hukushima,, and Hikaru Kawamura ${ }^{2,0}$ \\ 1 Department of Basic Science, University of Tokyo, \\ 3-8-1 Komaba, Meguro-ku, Tokyo 153-8902, Japan \\ 2 Department of Earth and Space Science, Faculty of Science, \\ Osaka University, Toyonaka, Osaka 560-0043, Japan
}

(Dated: November 11, 2017)

\begin{abstract}
Equilibrium properties of the three-dimensional isotropic Heisenberg spin glass are studied by extensive Monte Carlo simulations, with particular attention to the nature of its phase transition. A finite-size-scaling analysis is performed both for the spin-glass (SG) and the chiral-glass (CG) orders. Our results suggest that the model exhibits the $\mathrm{CG}$ long-range order at finite temperatures without accompanying the conventional SG long-range order, in contrast to some of the recent works claiming the simultaneous SG and CG transition. Typical length and time scales which represent a crossover from the spin-chirality coupling regime at short scales to the spin-chirality decoupling regime at long scales are introduced and examined in order to observe the true asymptotic transition behavior. On the basis of these crossover scales, discussion is given concerning the cause of the discrepancy between our present result and those of other recent numerical works.

PACS numbers: 75.10.Nr, 05.10.Ln,05.70.Fh,64.60.Fr
\end{abstract}

\section{INTRODUCTION}

Spin glasses (SGs) have attracted the attention of researchers both in experiments and theory as a prototype of complex systems with quenched randomness $\stackrel{\underline{\underline{1}}}{\mathrm{w}}$ SGs are random magnets in which magnetic ions interact with each other either ferromagnetically or antiferromagnetically, depending on their positions. Most of theoretical works have been so far devoted to the minimal SG model, i.e., the Ising Edwards and Anderson (EA) model. After a discussion early in the 1980s, it is now widely believed that a three-dimensional (3D) Ising SG model exhibits a SG phase transition at a finite temperature. Large-scale Monte Carlo (MC) simulations presented an evidence for the finite-temperature SG ordering ${ }^{2.3}$ Subsequently, the critical exponents evaluated by MC simulations were consistently compared with those evaluated experimentally for the Ising-like SG compound $\mathrm{FeMnTiO}_{3}$.

Compared to the Ising case, the nature of the phase transition of continuous spin systems such as XY and Heisenberg SGs are still poorly understood. Since many SG magnets including canonical SG possess only weak magnetic anisotropy, an isotropic Heisenberg SG model, rather than the strongly anisotropic Ising model, is expected to be a realistic model of SG magnets. Experimentally, an equilibrium SG phase transition has been established in real SG materials via measurements of the divergent non-linear susceptibility, etc. In sharp contrast to experiments, earlier theoretical studies on the Heisenberg SG model indicated that the standard SG long-range order occurred only at zero temperature in three dimensions 4.5 .6 .7 .8

In order to solve this apparent puzzle, a chirality mechanism of experimentally observed SG transitions was proposed by Kawamura.10. This scenario is based on the assumption that an isotropic 3D Heisenberg SG exhibits a finite-temperature chiral-glass (CG) transition without the conventional SG order. In terms of symmetry, among the global symmetries of the isotropic Hamiltonian, only the $Z_{2}$ spin-reflection (or spin-inversion) symmetry associated with the chirality is spontaneously broken with keeping the $S O(3)$ spin-rotation symmetry. Indeed, some numerical studies 11.12 claimed that the standard SG order associated with the freezing of the Heisenberg spin occurred at a temperature lower than the CG transition temperature, i.e., $T_{\mathrm{SG}}<T_{\mathrm{CG}}$, possibly with $T_{\mathrm{SG}}=0$. It means that the spin and the chirality are decoupled on long length and time scales, although the chirality is locally defined as a composite operator of the spin variables.

In this chirality scenario of experimental SG transitions, essential features of many of the real SG transition and of the SG ordered state are determined by the properties of the CG transition and of the CG state of the fully isotropic system. The role of the magnetic anisotropy is secondary which re-couples the spin to the chirality and reveals the CG transition in the chiral sector as an anomaly in experimentally accessible spin-related quantities. The scenario successfully explained the phase diagram under magnetic fields observed by the recent numerical simulation $\frac{13.14}{}$ and experiments $\stackrel{15}{\underline{15}}$

More recently, however, some researchers argued a possibility that in the 3D Heisenberg SG model the spin ordered at a finite temperature simultaneously with the chirality, i.e., $T_{\mathrm{SG}}=T_{\mathrm{CG}}>0.16 .17 .18 .19 .20$ Thus, the nature of the ordering of the 3D Heisenberg SG, as well as the validity of the chirality scenario, is now under debate. Under such circumstances, it is highly interesting to perform further extensive numerical studies of the 3D Heisenberg SG in order to clarify the true nature of its ordering. In the present study, we investigate both the SG and CG orderings of the model by means of a large-scale equilibrium MC simulation.

Interestingly, recent experiments reported on a quali- 
tative difference in aging phenomena between a canonical Heisenberg-like SG and an Ising-like SG $\stackrel{21}{\underline{1}}$ We also expect that the full understanding of the equilibrium properties of the 3D Heisenberg SG will also give a valuable insight into these off-equilibrium properties of SGs.

The article is organized as follows. In Sec. III we give a background of the present numerical study. In Sec. ПA we explain first the basics of the chirality mechanism. In Sec. II we introduce the crossover length and time scales beyond which the spin and the chirality are decoupled with each other. These length and time scales are crucially important in the chirality mechanism, and are also essential in properly interpreting the numerical data of MC simulations. In Sec. IIII we explain the model and the MC method employed. In Sec. IV] we introduce various physical quantities measured in our MC simulations, while the results of our simulations are presented in Sec. $\mathbf{D}$ In view of our MC results, we examine and discuss in Sec. VD the recent numerical results on the 3D Heisenberg SG by other authors. Finally, we present a brief summary of the results in section VII.

\section{BACKGROUND}

In this section, we wish to give a background of the present numerical study of the 3D isotropic Heisenberg SG. First, we explain the basics of the chirality mechanism of experimental SG transition as proposed in Refs. 9 and 10. Then, we explain the notion of the spin-chirality decoupling, together with the crossover length and time scales which play a crucially important role in the chirality mechanism and are also essential in properly interpreting the numerical data.

\section{A. Chirality mechanism}

Chirality is an Ising-like multi-spin variable representing the sense or the handedness of noncollinear spin structures induced by spin frustration. In frustrated magnets with continuous spins, the chirality often plays an essential role in their magnetic ordering. The local chirality $\chi_{i \mu}$ at the $i$ th site in the $\mu$-direction may be defined by

$$
\chi_{i \mu}=\vec{S}_{i+\hat{e}_{\mu}} \cdot\left(\vec{S}_{i} \times \vec{S}_{i-\hat{e}_{\mu}}\right),
$$

$\hat{e}_{\mu}(\mu=x, y, z)$ being a unit lattice vector along the $\mu$ axis. This quantity is often called a scalar chirality: It takes a non-zero value only when the three neighboring spins take the non-coplanar configuration in spin space, while it vanishes for the collinear or the coplanar spin configuration. The chirality defined above is a pseudoscalar variable since it is invariant under the global $S O(3)$ spin rotations but changes its sign under the global $Z_{2}$ spin reflections or inversions.
The chirality mechanism of Refs. 9 and 10 takes the following two-step strategy in explaining the real SG transition: The first step concerns with the property of the fully isotropic Heisenberg SG, an idealization of experimental SG materials. The chirality scenario claims that the fully isotropic Heisenberg SG exhibits a finite-temperature CG transition without the conventional SG long-range order. The CG transition breaks only the $Z_{2}$ spin-reflection symmetry with keeping the $S O(3)$ spin-rotational symmetry. The occurrence of the CG transition necessarily entails the spin-chirality decoupling.

Obviously, such a scenario does not apply to the infinite dimensional limit, i.e., to the mean-field Heisenberg Sherrington-Kirkpatrick (SK) model, in which the spin itself, not the chirality, behaves as an order parameter of the transition. Due to the noncoplanar nature of the spin configuration in the SG state, the SG long-range order trivially accompanies the CG long-rage order, whereas the opposite is not necessarily true. One should note that, in the conventional case where the spin variable is a proper order parameter of the transition as in the case of the SK model, the chirality, which is given by the multiple of the spin, exhibits a less singular behavior than the spin at the SG transition. In fact, the chirality shows only moderate behavior at the SG transition of the mean-field Heisenberg SK model in which the spin, not the chirality, is the order parameter of the transition ${ }^{22}$

In contrast to the mean-field model or the highdimensional Heisenberg SG models, the problem could be very non-trivial in lower dimensions where the orderparameter fluctuation might change the nature of ordering dramatically. At present, there seems to be no consensus about the lower critical dimension $d_{\mathrm{SG}}^{\mathrm{LCD}}$ of the $\mathrm{SG}$ order, while the corresponding upper critical dimension is expected to be six. The CG order, if exists, may emerge slightly above, at, or below $d_{\mathrm{SG}}^{\mathrm{LCD}}$.

It has been proved that the SG long-range order does not exist at any finite temperature in the two dimensional Heisenberg SG $\stackrel{23}{?}$ The numerical domain-wall renormalization-group calculation as well as the MC simulation suggested that both the spin and the chirality ordered only at zero temperature ${ }^{\frac{9}{}}$. Interestingly, however, the estimated SG and CG correlation-length exponents at this $T=0$ transition differ significantly from each other, i.e., $\nu_{\mathrm{CG}}>\nu_{\mathrm{SG}} \stackrel{24}{2}$ This implies that in $2 \mathrm{D}$ the spin and the chirality are decoupled at long length scale, the chirality dominating the long-length behavior.

In view of these transition behaviors of the Heisenberg SGs, it appears likely that the principal player in longscale phenomena changes from the spin to the chirality as the spacial dimensionality is decreased. Thus, the behavior in dimension three is the current issue, which is the subject of the present work.

The second step of the chirality mechanism concerns with the effect of the random magnetic anisotropy which inevitably exists in real SG magnets. The random anisotropy energetically breaks the $S O(3)$ spin-rotation symmetry in the Hamiltonian, retaining the $Z_{2}$ inversion 


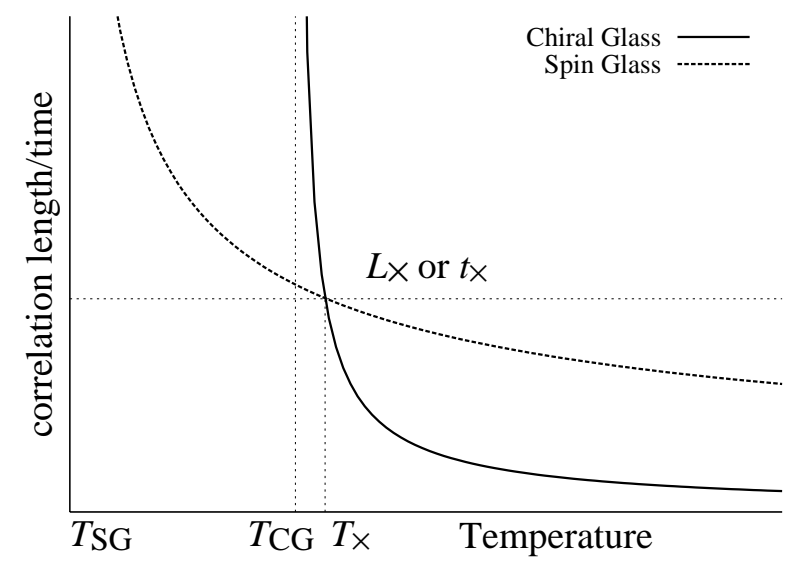

FIG. 1: A schematic figure of the crossover between the spinglass and chiral-glass correlation lengths (correlation times) expected in the chirality mechanism. According to the chirality mechanism, the CG correlation length (correlation time) diverges toward the CG transition temperature $T=T_{\mathrm{CG}}$, while the $\mathrm{SG}$ one diverges at a lower temperature $T=T_{\mathrm{SG}}<$ $T_{\mathrm{CG}}$.

symmetry only. When the anisotropic system exhibits the CG long-range order with spontaneously breaking the $Z_{2}$ inversion symmetry, there no longer remains any global symmetry degree of freedom to leave the system in the paramagnetic phase. Hence, once the CG order occurs in the presence of the random anisotropy, the spin degree of freedom also behaves like the chirality. This is the spin-chirality recoupling due to the random magnetic anisotropy.

Such an anomaly revealed in the spin sector via the random magnetic anisotropy can be detected experimentally by standard magnetic measurements e.g. , as a divergence of the nonlinear susceptibility etc, whereas the CG long-range order is difficult to observe experimentally.

We note that in this mechanism the anisotropy plays only a secondary role: The anisotropy certainly reduces the symmetry of the Hamiltonian relative to the fully isotropic system, but does not change the broken symmetry of the transition. The critical properties of the CG transition and of the low-temperature CG phase are expected to be not affected by the magnetic anisotropy, which, however, are now directly observable via the standard spin-related quantities. This chirality scenario predicts that experimentally observed SG transitions belong to the same universality class as that of the the CG transition of the fully isotropic model. It is thus highly interesting to clarify the nature of the phase transition of the ideal isotropic Heisenberg SG.

\section{B. Spin-chirality decoupling/coupling scenario}

As mentioned above, in lower dimensions, a relevant degree of freedom which dominates the long-scale phenomena might well change from the spin to the chirality. The chirality scenario expects that in $3 \mathrm{D}$ there exists a crossover temperature $T_{\times}$which separates the two temperature regimes, as illustrated in Fig. 1] In the higher temperature regime, the SG correlation length is longer than the CG correlation length, dominating the longscale phenomena. This is simply due to the fact that the sensible definition of the local chirality requires the development of the spin short-range order of at least a few lattice spacings. As the temperature is decreased, both the SG and CG correlation lengths grow, but at different rates, so that the $\mathrm{CG}$ correlation length eventually outgrows the SG correlation length at the crossover temperature $T_{\times}$. An example of such a crossover behavior between the spin and the chiral correlations can be seen explicitly in a certain toy model: See Fig. 10 of Ref. 25. Then, the relevant degree of freedom for the long-scale behavior changes at $T_{\times}$from the spin to the chirality. Below $T_{\times}$, the long-scale phenomena are governed by the CG correlation, not by the SG one. This is the spin-chirality decoupling expected to occur in the fully isotropic model.

Let us discuss in some detail the finite-size effect inherent to the simulation data in the critical region. The situation here is not simple because the system has two length scales, each associated with the spin and with the chirality. Suppose that the CG transition occurs at $T=T_{\mathrm{CG}}$ without the conventional SG long-range order. Then, the crossover temperature $T_{\times}$at which the CG correlation length outgrows the SG correlation length should be located somewhat above $T_{\mathrm{CG}}$ : See Fig.1. A necessary condition for detecting the spin-chirality decoupling is that the measurement temperature lies below $T_{\times}$. It is, however, not enough. At a temperature below $T_{\times}$, one needs to probe the system beyond the crossover length above which the spin-chirality decoupling becomes eminent. Thus, a large-size simulation exceeding the crossover length is required in order to detect the spin-chirality decoupling. Unfortunately, the crossover length scale is unknown a priori, and is to be investigated by numerical simulations. A natural criterion might be that it is given by the SG correlation length at the crossover temperature $T_{\times}$as shown in Fig. 1] Even in the $\mathrm{CG}$ ordered phase, the spin-chirality decoupling might hardly be observable at the length scale below the crossover length. Rather, it is natural to expect that the trivial spin-chirality coupling is observed below the crossover length scale because the chirality is a composite operator of the spin on the short scale of lattice spacing not independent of the spin, roughly being $\chi \approx S^{3}$.

In the CG critical region, the chirality-related quantities should exhibit the true asymptotic critical behavior, e.g., a power-law singularity characterized by the associated CG exponents. At short length scales below the 


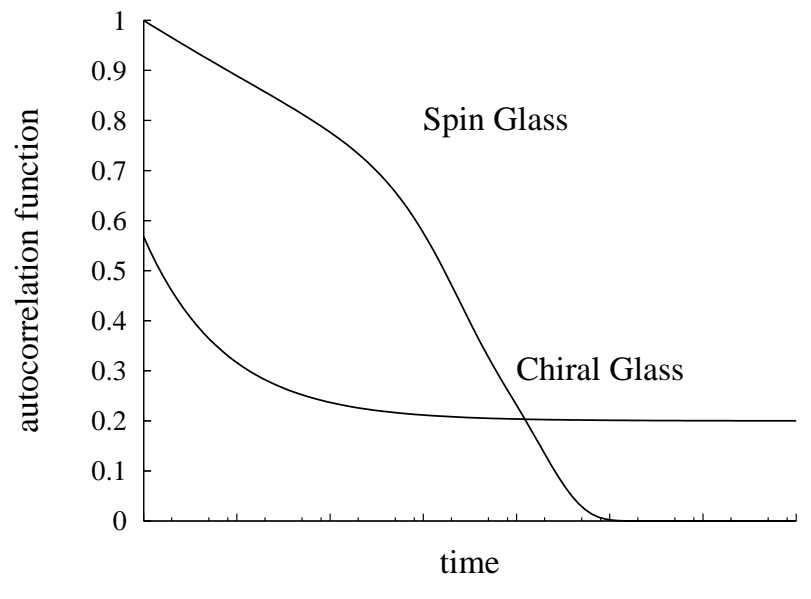

FIG. 2: A schematic figure of the time evolution of the spinglass and chiral-glass two-time autocorrelation functions.

crossover length scale, due to the trivial coupling between the spin and the chirality, even the spin-related quantities are expected to exhibit the similar critical behavior to the chirality-related quantities. Namely, up to the crossover length scale, it seems as if the SG order developed as a long-range order. It is intrinsically difficult at shorter length scales to distinguish such a pseudo-critical behavior induced by the CG long-range order from the true SG long-range order. Hence, it is crucially important to estimate the crossover length scale and to study the long-scale behavior of the system beyond this length scale.

Essentially the same argument also applies to the case of the temporal scale. As an example, we discuss here the behavior of the autocorrelation functions based on the notion of the crossover time scale. Figure 2 shows the schematic representation of the behavior of the SG and $\mathrm{CG}$ autocorrelation functions below $T_{\mathrm{CG}}$ expected from the spin-chirality coupling/decoupling picture. In the long-time limit, the chirality autocorrelation function is expected to saturate to a certain finite value after an initial fast decay, while the spin autocorrelation function is expected to decay toward zero asymptotically. A comment is in order concerning the transient behavior of the spin autocorrelation function at time scales shorter than the crossover time scale: At shorter time scales, the spin autocorrelation function might exhibit the pseudoordering feature dictated by the CG one through the trivial spin-chirality coupling. This might well lead to a hump-like pseudo-ordering structure in the time dependence of the spin autocorrelation function as shown in Fig.2, which, however, does not persist in the long-time limit beyond the crossover time scale. This means that, in order to properly discuss the true asymptotic behavior of the dynamics of the model, a particular attention should be paid to the crossover time scale.

\section{THE MODEL AND THE MONTE CARLO METHOD}

We study a classical Heisenberg model defined by the Hamiltonian,

$$
H(\vec{S})=-\sum_{\langle i j\rangle} J_{i j} \vec{S}_{i} \cdot \vec{S}_{j}
$$

where $\vec{S}_{i}=\left(S_{i}^{x}, S_{i}^{y}, S_{i}^{z}\right)$ is a three-component unit vector, and the summation runs over all nearest-neighbor pairs. The lattice is a simple-cubic lattice with the total number of $N=L^{3}$ sites. The nearest-neighbor couplings $J_{i j}$ take the values $\pm J$ randomly with equal probability. Periodic boundary conditions are imposed for all the directions. The lattice sizes studied are $L=8,12,16$ and 20, where the sample average is taken over $976(L=8), 964(L=$ 12), $280(L=16)$, and $32(L=20)$ independent bond realizations.

We perform an equilibrium MC simulation of the model. In our simulation, we make use of the exchange MC method, $\stackrel{26}{r}$ which is also called parallel tempering $\stackrel{27}{ }$ In the exchange MC method, one MC step consists of two elementary updates, a standard single-spin heat-bath flip ${ }^{6}$ and an exchange trial of spin configurations at neighboring temperatures. The latter reduces the slow relaxation at low temperatures with the help of the hightemperature fast dynamics. The method has turned out to be quit efficient in thermalizing a wide class of hardlyrelaxing systems such as SG systems and proteins. We ensure equilibration by checking that various observables attain stable values, no longer changing with the amount of MC steps: See Refs. 22 and 28 for further details of the equilibration procedure. Our MC simulations have been performed up to the size $L=20$ and up to the temperature $T / J=0.15$. This could be achieved only by using the exchange MC method. The numbers of temperature points used in our exchange MC method are 32 for $L=8,12,16$, and 48 for $L=20$.

Error bars are estimated via sample-to-sample fluctuations for the linear quantities such as the order parameters, and by the jackknife method for the non-linear quantities such as the Binder parameter and the correlation length mentioned below.

\section{PHYSICAL OBSERVABLES}

In the present section, we introduce various physical quantities observed in our simulations, and discuss some of their basic properties.

In glassy systems, it is often convenient to define as an order parameter an overlap variable between two independent systems with the same Hamiltonian. For the Heisenberg spin, the overlap may be defined as a tensor variable between the $\mu$ and $\nu$ components $(\mu, \nu=x, y, z)$ 
of the Heisenberg spin by

$$
q_{\mu \nu}=\frac{1}{N} \sum_{i=1}^{N} S_{i \mu}^{(1)} S_{i \nu}^{(2)},
$$

where the upper suffixes (1) and (2) denote the two replicas of the system with the same interaction set.

A chiral overlap is defined in terms of the local chiral variable (1) by

$$
q_{\chi}=\frac{1}{3 N} \sum_{i \mu} \chi_{i \mu}^{(1)} \chi_{i \mu}^{(2)} .
$$

The squared SG order parameter is then given by

$$
q_{\mathrm{SG}}^{(2)}=\left[\left\langle\sum_{\mu \nu} q_{\mu \nu}^{2}\right\rangle\right],
$$

where $\langle\cdots\rangle$ denotes a thermal average and $[\cdots]$ denotes an average over the bond disorder. The corresponding squared $\mathrm{CG}$ order parameter is defined by

$$
q_{\mathrm{CG}}^{(2)}=\frac{\left[\left\langle q_{\chi}^{2}\right\rangle\right]}{\bar{\chi}^{4}},
$$

which is normalized by the mean-square amplitude of the local chirality,

$$
\bar{\chi}^{2}=\frac{1}{3 N} \sum_{i}^{N} \sum_{\mu}\left[\left\langle\chi_{i \mu}^{2}\right\rangle\right] .
$$

The local chirality amplitude remains non-zero only when the spins have a non-coplanar structure locally. This quantity weakly depends on the temperature, in contrast to the Heisenberg spin variable whose amplitude is fixed to be unity by definition. In the high-temperature symmetric phase, these SG and $\mathrm{CG}$ order parameters are essentially equivalent to the associated SG and CG susceptibilities defined by $\chi_{\mathrm{SG}}=N q_{\mathrm{SG}}^{(2)}$ and $\chi_{\mathrm{CG}}=3 N q_{\mathrm{CG}}^{(2)}$, respectively.

A standard finite-size scaling of the second-order transition for the equilibrium SG and CG order parameters takes the form

$$
q^{(2)} \sim L^{-(1+\eta)} f\left(\left|T-T_{\mathrm{c}}\right| L^{1 / \nu}\right),
$$

where $\nu$ is the exponent of the correlation length, and $\eta$ is the exponent describing the decay of the correlation function at the critical point $T=T_{\mathrm{c}}$. At $T_{\mathrm{c}}$, the order parameter decays as a power law with the size $L$,

$$
q^{(2)} \propto L^{-(1+\eta)} .
$$

One often uses the Binder parameter to estimate the critical temperature. In the Heisenberg SG, the Binder parameter for the SG order is defined by

$$
g_{\mathrm{SG}}=\frac{1}{2}\left(11-9 \frac{\left[\left\langle q^{4}\right\rangle\right]}{\left[\left\langle q^{2}\right\rangle\right]^{2}}\right), \quad q^{2}=\sum_{\mu, \nu} q_{\mu \nu}^{2}
$$

while that for the CG oder is defined by

$$
g_{\mathrm{CG}}=\frac{1}{2}\left(3-\frac{\left[\left\langle q_{\chi}^{4}\right\rangle\right]}{\left[\left\langle q_{\chi}^{2}\right\rangle\right]^{2}}\right) .
$$

In the thermodynamic limit, these Binder parameters are normalized to unity in the non-degenerate ordered state, and to zero in the high-temperature disordered state. Since the Binder parameter is a dimensionless quantity, and the dimensionless quantity should be sizeindependent at the critical temperature $T_{c}$, the Binder parameters of different system sizes plotted as a function of temperature should yield a crossing or merging point at $T_{c}$.

In terms of the $k$-dependent overlap variable, one can define the Fourier-transformed two-point CG and SG correlation functions. For the $\mathrm{CG}$, the $k$-dependent chiraloverlap is defined by

$$
q_{\chi}(\vec{k})=\frac{1}{N} \sum_{i=1}^{N} \chi_{i x}^{(1)} \chi_{i x}^{(2)} \exp \left(i \vec{k} \cdot \vec{r}_{i}\right)
$$

in which the chiral variable along the $x$-axis, $\chi_{i x}$, is considered. The Fourier-transformed CG correlation function is then defined by

$$
q_{\mathrm{CG}}^{(2)}(\vec{k})=\left[\left\langle\left|q_{\chi}(\vec{k})\right|^{2}\right\rangle\right] .
$$

For the $\mathrm{SG}$, the $k$-dependent spin-overlap is defined by

$$
q_{\mu \nu}(\vec{k})=\frac{1}{N} \sum_{i=1}^{N} S_{i \mu}^{(1)} S_{i \nu}^{(2)} \exp \left(i \vec{k} \cdot \vec{r}_{i}\right),
$$

whereas the Fourier-transformed SG correlation function is defined by

$$
q_{\mathrm{SG}}^{(2)}(\vec{k})=\left[\left\langle\sum_{\mu \nu}\left|q_{\mu \nu}(\vec{k})\right|^{2}\right\rangle\right] .
$$

Via these CG and SG Fourier-transformed correlation functions, the associated CG and SG finite-system correlation lengths are defined by

$$
\xi=\frac{1}{2 \sin \left(k_{\mathrm{m}} / 2\right)} \sqrt{\frac{q^{(2)}(\overrightarrow{0})}{q^{(2)}\left(\vec{k}_{\mathrm{m}}\right)}-1},
$$

where $\vec{k}_{\mathrm{m}}=(2 \pi / L, 0,0)$ and $k_{\mathrm{m}}=\left|\vec{k}_{\mathrm{m}}\right|$.

One can then define a dimensionless quantity, the normalized correlation length $\xi_{\mathrm{CG}} / L$ and $\xi_{\mathrm{SG}} / L$. Since $\xi / L$ is dimensionless, it should exhibit the same scaling property as the Binder parameter. Thus, the ratio $\xi / L$ for different $L$ should cross or merge at the critical temperature.

In probing the nature of the low-temperature glassy ordered phase, one useful quantity is the distribution of 
the overlap. The chiral-overlap distribution is defined by

$$
P\left(q_{\chi}^{\prime}\right)=\left[\left\langle\delta\left(q_{\chi}^{\prime}-q_{\chi}\right)\right\rangle\right] .
$$

The squared CG order parameter $q_{\mathrm{CG}}^{(2)}$ defined above is the second moment of the chiral-overlap distribution function.

The spin-overlap distribution is defined originally in the tensor space with $3 \times 3=9$ components. To make the quantity more easily tractable, one may define the diagonal spin overlap which is the trace of the original tensor overlap, and introduce the associated diagonalspin-overlap distribution by

$$
P\left(q_{\text {diag }}\right)=\left[\left\langle\delta\left(q_{\text {diag }}-\left(\sum_{\mu=x, y, z} q_{\mu \mu}\right)\right)\right\rangle\right] .
$$

This distribution function is symmetric with respect to $q_{\text {diag }}=0$, and is expected to be a Gaussian distribution around $q_{\mathrm{diag}}=0$ in the high-temperature disordered phase. Reflecting the fact that the diagonal-spinoverlap is not invariant under the global $O(3)$ spin rotation, $P\left(q_{\text {diag }}\right)$ in the possible SG ordered phase develops a nontrivial shape, not just consisting of the delta-function peaks related to $q_{\mathrm{EA}}$, even when the ordered state is a trivial one simply described by a self-overlap $q_{\mathrm{EA}}{ }^{22}$ If the possible SG ordered state accompanies a replicasymmetry breaking (RSB), further nontrivial structures would be added to $P\left(q_{\text {diag }}\right)$. Meanwhile, it is recently shown in Ref. 22 that, in the possible SG ordered state, the diverging peaks corresponding to the self-overlap necessarily appears in $P\left(q_{\text {diag }}\right)$ at $q_{\text {diag }}= \pm \frac{1}{3} q_{\mathrm{EA}}$ in the thermodynamic limit. Hence, the existence or nonexistence of these divergent peaks could be used as an unambiguous measure of the possible SG long-range order in the Heisenberg SG, irrespective the occurrence of the RSB.

Another interesting feature of glassy systems might be the nature of their sample-to-sample fluctuations, particularly its possible non-self-averageness. As an indicator of the lack of self-averageness, one may use the so-called $A$ parameter ${ }^{29}$ For the CG order, it is defined by

$$
A_{\mathrm{CG}}(T) \equiv \frac{\left[\left\langle q_{\chi}^{2}\right\rangle^{2}\right]-\left[\left\langle q_{\chi}^{2}\right\rangle\right]^{2}}{\left[\left\langle q_{\chi}^{2}\right\rangle\right]^{2}}
$$

while for the SG order,

$$
A_{\mathrm{SG}}(T) \equiv \frac{\left[\left\langle q^{2}\right\rangle^{2}\right]-\left[\left\langle q^{2}\right\rangle\right]^{2}}{\left[\left\langle q^{2}\right\rangle\right]^{2}} .
$$

The order parameter is non-self-averaging when the associated $A$ parameter is non-zero, and is self-averaging when $A$ is equal to zero.

One can also define the so-called Guerra parameter $G \stackrel{30}{\underline{30}}$ For the CG order, it is defined by

$$
G_{\mathrm{CG}}(T) \equiv \frac{\left[\left\langle q_{\chi}^{2}\right\rangle^{2}\right]-\left[\left\langle q_{\chi}^{2}\right\rangle\right]^{2}}{\left[\left\langle q_{\chi}^{4}\right\rangle\right]-\left[\left\langle q_{\chi}^{2}\right\rangle\right]^{2}}
$$

while for the SG order,

$$
G_{\mathrm{SG}}(T) \equiv \frac{\left[\left\langle q^{2}\right\rangle^{2}\right]-\left[\left\langle q^{2}\right\rangle\right]^{2}}{\left[\left\langle q^{4}\right\rangle\right]-\left[\left\langle q^{2}\right\rangle\right]^{2}} .
$$

Unlike the $A$ parameter, the $G$ parameter can take a non-zero value even when the ordered state is a trivial one without accompanying the RSB ${ }^{31.32}$ The $G$ parameters are related to the $A$ parameters and the Binder parameters $g$ via the relations,

$$
\begin{gathered}
A_{\mathrm{CG}}=2\left(1-g_{\mathrm{CG}}\right) G_{\mathrm{CG}}, \\
A_{\mathrm{SG}}=\frac{2}{9}\left(1-g_{\mathrm{SG}}\right) G_{\mathrm{SG}} .
\end{gathered}
$$

These relations indicate that, so long as the Binder parameter $g$ takes any value different from unity in the ordered phase, a non-zero $A$ necessarily means a non-zero $G$. By contrast, if the Binder parameter $g$ takes a value unity in the ordered phase, a non-zero $A$ may or may not mean a non-zero $G$.

Information about the equilibrium dynamics can be obtained from the spin and chiral two-time autocorrelation functions defined by

$$
\begin{aligned}
& C_{s}(t)=\frac{1}{N} \sum_{i}\left[\left\langle\vec{S}_{i}\left(t_{0}\right) \cdot \vec{S}_{i}\left(t+t_{0}\right)\right\rangle\right], \\
& C_{\chi}(t)=\frac{1}{3 N} \sum_{i \mu}\left[\left\langle\chi_{i \mu}\left(t_{0}\right) \chi_{i \mu}\left(t+t_{0}\right)\right\rangle\right],
\end{aligned}
$$

where the time evolution in our MC simulation is made according to the standard heat-bath updating not accompanying the exchange process. Initial spin configurations at $t=t_{0}$ are taken from equilibrium spin configurations generated in our exchange MC runs. Below the transition temperature $T_{c}$ (if any), these autocorrelation functions converge in the long time limit to the Edwards-Anderson SG and CG order parameters, whereas above $T_{c}$ these autocorrelation functions decay exponentially toward zero with a characteristic correlation time, which diverges as the temperature $T$ approaches $T_{c}$. Just at $T_{c}$, the autocorrelation functions exhibit a power-law decay,

$$
C(t) \sim t^{-\beta / z \nu},
$$

where $z$ is the dynamical critical exponent. These features are described by the standard bulk dynamical scaling form,

$$
C(t) \sim\left|T-T_{\mathrm{c}}\right|^{\beta} f\left(t\left|T-T_{\mathrm{c}}\right|^{z \nu}\right),
$$

where $f(x)$ is a scaling function whose asymptotic forms for $x \ll 1$ and $x \gg 1$ are $x^{-\beta / z \nu}$ and $\exp (-x)$, respectively.

\section{THE NUMERICAL RESULTS}

In the present section, we show our MC results of the three-dimensional isotropic Heisenberg SG model. 

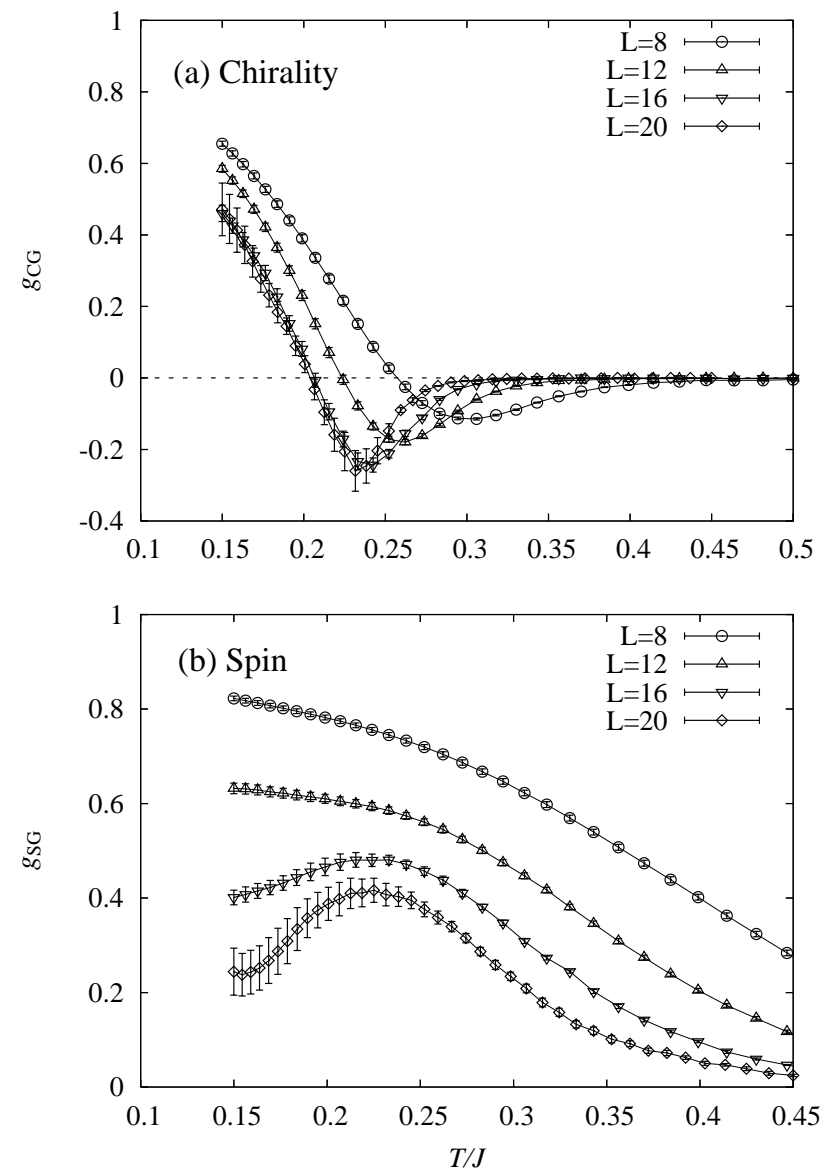

FIG. 3: The temperature and size dependence of the chiralglass Binder parameter; upper figure (a), and of the spin-glass Binder parameter; lower figure (b), of the $3 \mathrm{D} \pm J$ Heisenberg SG.

\section{A. Binder parameter}

In Fig. 3 we show the temperature and size dependence of the Binder parameters both for the chirality; upper figure (a), and for the spin; lower figure (b). As can be seen from Fig. [3(a), a crossing of the CG Binder parameter $g_{\mathrm{CG}}$ of different $L$ is observed on the negative side of $g_{\mathrm{CG}}$, not on the positive side as in the standard cases. With increasing $L$, the crossing temperature gradually shifts toward lower temperatures. A behavior similar to this has also been observed in the Binder parameter of other models, including the Heisenberg SG ${ }^{12,22}$ and the mean-field $\mathrm{SG}^{33.34}$. In particular, in a class of mean-field SG models exhibiting a one-step RSB, the Binder parameter at the transition point $T_{\mathrm{c}}$ takes a negative value, sometimes even negatively divergent 33.34 It implies that the temperature at which the Binder parameter for finite $L$ takes a minimum, a dip temperature $T_{\text {dip }}(L)$, approaches the critical temperature, i.e., $T_{\text {dip }}(L) \rightarrow T_{\text {c }}$ as $L \rightarrow \infty$. Recently, this method of estimating the bulk transition temperature was successfully applied to the Heisenberg SG $\stackrel{13,22}{=}$ In Fig. [u we plot

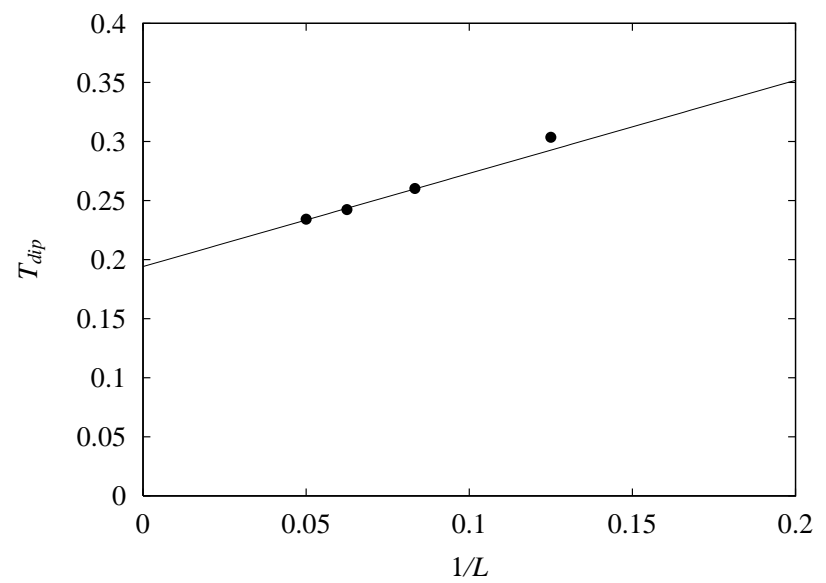

FIG. 4: The dip temperature of the chiral-glass Binder parameter $g_{\mathrm{CG}}$ is plotted against $1 / L$. The solid line represents a linear fit of the data. Its extrapolation to the $L \rightarrow \infty$ limit gives an estimate of the bulk chiral-glass transition temperature, $T_{\mathrm{CG}} / J=0.194(5)$.

$T_{\text {dip }}(L)$ against $1 / L$. An extrapolation to the thermodynamic limit $1 / L \rightarrow 0$ gives us an estimate of the bulk CG transition temperature, $T_{\mathrm{CG}} / J=0.194(5)$.

By contrast, as shown in Fig. 3(b), the SG Binder parameter $g_{\mathrm{SG}}$ monotonically decreases toward zero with increasing $L$ at all temperatures studied. There is no signature of the transition in the investigated temperature range, no negative dip nor the crossing, in contrast to the CG Binder parameter. This suggests that the SG transition temperature, if any, is located at a temperature lower than the temperature range studied here. Fig. B(b) reveals, however, that an anomalous bend appears in $g_{\mathrm{SG}}$ for larger sizes $L \geq 16$ at around $T / J \simeq 0.22$, close to the CG transition temperature, although $g_{\mathrm{SG}}$ never becomes size-invariant at any temperature, as it should have been in a second-order transition. The reason why $g_{\mathrm{SG}}$ exhibits such an anomalous bend around $T_{\mathrm{CG}}$ might be understood as follows: At the CG transition, a reflection symmetry is spontaneously broken and the entire phase space is divided into ergodic components, in each of which a proper-rotational symmetry is still preserved. As a result, the ordering behavior of the Heisenberg spin would change at $T_{\mathrm{CG}}$, though the spin itself does not order even below $T_{\mathrm{CG}}$. We note that a similar bend in $g_{\mathrm{SG}}$ has also been observed in the two-dimensional Heisenberg SG 24 where the absence of a finite-temperature SG transition has been well established ${ }^{23}$

It is sometimes argued in the literature that the Binder-parameter analysis might not work in the SG problem. Such a suspicion might partly be based on the observation that only weak merging behavior was observed at the SG transition temperature of the threedimensional EA Ising model which is believed to exhibit a finite-temperature SG transition 2.2 .35 As long as the SG long-rage order really sets in at finite temperatures, however, it is hardly conceivable that the Binder pa- 
rameter for asymptotically large lattices exhibits a nonsingular behavior only. In particular, the Binder parameter should become scale-invariant at the SG transition point, so long as the transition is continuous. Indeed, in a recent $\mathrm{MC}$ simulation of the mean-field Heisenberg $\mathrm{SG}^{22}$ which is known to exhibit a non-zero SG long-range order below $T_{\mathrm{SG}}$, a clear crossing of the SG Binder parameter $g_{\mathrm{SG}}$ has been observed at $T_{\mathrm{SG}}$, in sharp contrast to our present data of Fig. 3(b).

Then, one might argue that the finite-size effect would be significant here in $g_{\mathrm{SG}}$ and the large- $L$ asymptote might still be far away. One sees from Fig. 3(a), however, that the $\mathrm{CG}$ Binder parameter $g_{\mathrm{CG}}$ for our two largest sizes $L=16$ and 20 exhibits an almost size-invariant behavior at and below $T_{\mathrm{CG}}$. If the Heisenberg spin orders simultaneously with the chirality, and if the spin is the order parameter of the transition and the chirality is only composite (secondary), it seems a bit hard to understand why the chirality exhibits an almost scale-invariant near-critical behavior for $L \geq 16$, while the Heisenberg spin still exhibits an off-critical scale-dependent behavior. Hence, the behavior of $g_{\mathrm{SG}}$ observed in Fig. 3(b) remains to be resolved if the occurrence of a finite-temperature SG transition is to be accepted in the investigated temperature range.

\section{B. Order parameter}

In Fig. 5 we show the size dependence of the squared $\mathrm{CG}$ and SG order parameters, $q_{\mathrm{CG}}^{(2)}$ and $q_{\mathrm{SG}}^{(2)}$, for various temperatures. In the upper figure (a), a doublelogarithmic plot of the CG order parameter $q_{\mathrm{CG}}^{(2)}$ is shown against the system size $L$. One generally expects that at $T_{c}$ the data of $q_{\mathrm{CG}}^{(2)}$ should lie on a straight line. In fact, the data of $q_{\mathrm{CG}}^{(2)}$ show a clear straight-line behavior around $T / J=0.19$, which is close to the CG transition temperature obtained by our analysis of $g_{\mathrm{CG}}$. The critical-point decay exponent $\eta_{\mathrm{CG}}$ can be estimated from the slope of this straight line, yielding $1+\eta_{\mathrm{CG}} \sim 1$.8. At higher temperatures, a deviation from the straight line, a downward trend, is observed indicative of the disordered phase. At lower temperatures, particularly at our lowest temperature simulated $T / J=0.15$, the data of $q_{\mathrm{CG}}^{(2)}$ show a clear upward trend. This suggests that this temperature is indeed below $T_{\mathrm{CG}}$, and that the low-temperature phase is a rigid one characterized by a non-zero $q_{\mathrm{CG}}^{(2)}$, not likely to be a critical phase like the Kosterlitz-Thouless (KT) phase.

In Fig. 5(b), a double-logarithmic plot of the corresponding SG order parameter $q_{\mathrm{SG}}^{(2)}$ is shown against the system size $L$. Again, the data are expected to lie on a straight line at the critical SG transition temperature, if any. Such a straight-line behavior, however, is found only at our lowest temperature studied $T / J=0.15$, whereas $q_{\mathrm{SG}}^{(2)}$ never exhibits an upward trend characteristic of the
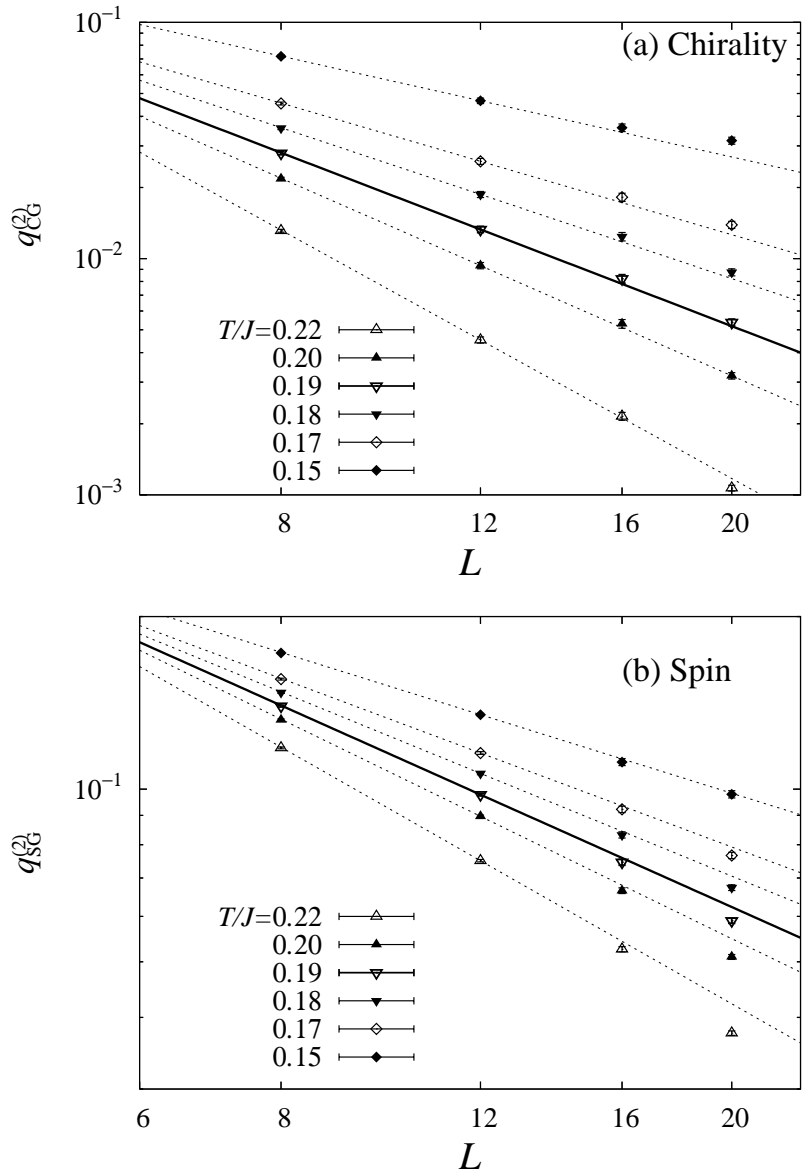

FIG. 5: Double-logarithmic plot of the squared chiral-glass order parameter $q_{\mathrm{CG}}^{(2)}$; upper figure (a), and of the squared spin-glass order parameter $q_{\mathrm{SG}}^{(2)}$; lower figure (b), as a function of the system size $L$ for several temperatures around the expected chiral-glass transition temperature. Straight lines are drawn by connecting the two data points of $L=8$ and 12 at each temperature.

long-range ordered phase at any temperature studied, in sharp contrast to the behavior of $q_{\mathrm{CG}}^{(2)}$. At temperatures higher than $T / J=0.15$, including the one at the $\mathrm{CG}$ transition temperature $T / J \simeq 0.19$, the data of $q_{\mathrm{SG}}^{(2)}$ show a linear behavior for smaller sizes, which gradually changes into a downward trend for larger sizes. This can simply be interpreted as a size-crossover which occurs around the length scale of the SG correlation length at each temperature. We note that such a size-crossover is clearly discernible even at a temperature $T / J=0.17$ which is below $T_{\mathrm{CG}}$. The length scale of the crossover, comparable to the spin correlation length, grows as $T$ decreases, and it is considered to exceed our largest size $L=20$ at around $T / J=0.15$.

This observation strongly suggests that the standard SG transition temperature of the model is lower than $T / J=0.15$, and that, at least within the temperature range $0.15 \lesssim T \lesssim 0.19$, solely the CG long-range order 


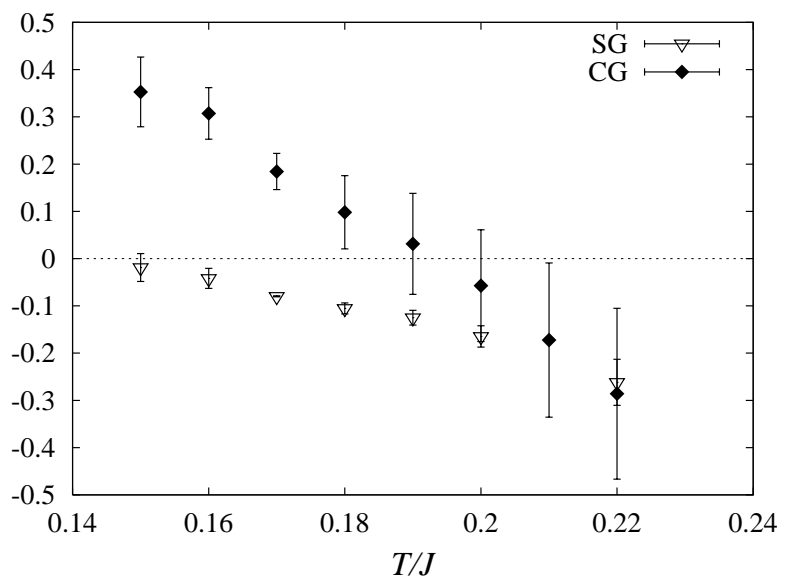

FIG. 6: The curvatures of the $L$-dependence of the squared spin-glass order parameter $q_{\mathrm{SG}}^{(2)}$ (open symbols), and of the squared chiral-glass order parameter $q_{\mathrm{CG}}^{(2)}$ (filled symbols), are plotted versus the temperature. The curvature is expected to be zero at the respective transition temperature.

exists without the standard SG long-range order, i.e., one has $T_{\mathrm{CG}}>T_{\mathrm{SG}}$.

To make the situation more pronounced, we estimate following Refs. 36 and 37 the curvatures of the $L$ dependence of the two order parameters, $q_{\mathrm{SG}}^{(2)}$ and $q_{\mathrm{CG}}^{(2)}$, via second-order polynomial fits to the data of Figs. [5] The curvature is expected to be zero at the respective transition temperature. As shown in Fig. 6] the curvature for the $\mathrm{CG}$ crosses the zero-axis around $T / J \simeq 0.19$, while that for the SG does not cross the zero-axis there, but marginally touches on it at a lower temperature, $T / J \simeq 0.15$. The result indicates that the two transition temperatures, $T_{\mathrm{SG}}$ and $T_{\mathrm{CG}}$, are well separated.

\section{Finite-size scaling of the order parameter}

In order to estimate the correlation-length exponent $\nu$ associated with the CG transition, we apply the standard finite-size scaling analysis to the squared CG order parameter $q_{\mathrm{CG}}^{(2)}$ based on Eq. (8). By taking $\mid T-$ $T_{\mathrm{CG}} \mid / T_{\mathrm{CG}} L^{1 / \nu}$ as the scaling variable, the best data collapse is obtained with $T_{\mathrm{CG}} / J=0.19, \nu_{\mathrm{CG}}=1.2$ and $\eta_{\mathrm{CG}}=0.8$. As shown in Fig. 7 the data both below and above $T_{\mathrm{CG}}$ scale fairly well. If $\left|1 / T-1 / T_{\mathrm{CG}}\right| T_{\mathrm{CG}} L^{1 / \nu}$ is taken as the scaling variable, on the other hand, a slightly larger value of $\nu$, i.e., $\nu_{\mathrm{CG}}=1.4$ and $\eta_{\mathrm{CG}}=0.7$, is preferred. The observed difference in the best values of the exponents might be due to the correction to scaling. Thus, we finally quote $T_{\mathrm{CG}} / J=0.19(1), \nu_{\mathrm{CG}}=1.3(2)$ and $\eta_{\mathrm{CG}}=0.8(2)$. The error bar is estimated by examining the quality of the fits with varying the scaling parameters. The estimated values of critical exponents are compatible with the previous values obtained for the Heisenberg SG model but with the Gaussian bond

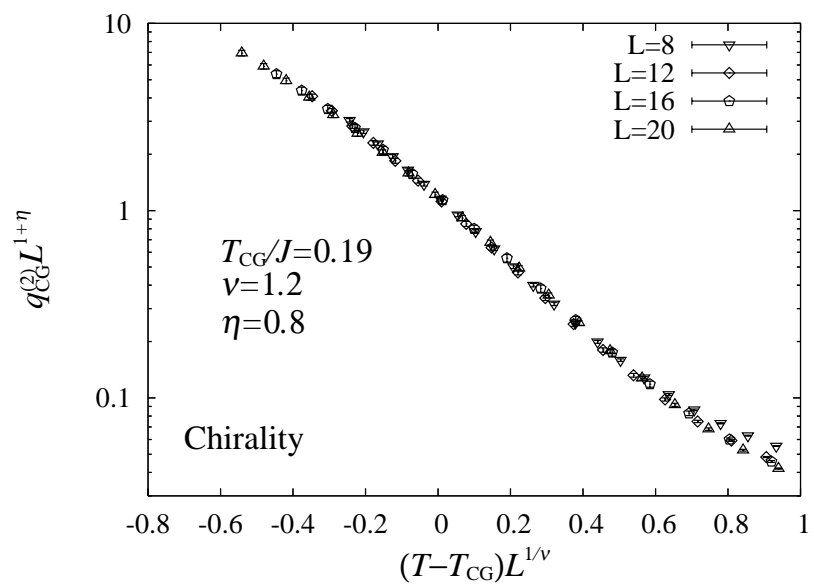

FIG. 7: Finite-size-scaling plot of the squared chiral-glass order parameter. The best scaling is obtained with $T_{\mathrm{CG}} / J=$ $0.19, \nu=1.2$ and $1+\eta=1.8$.

distribution, 12 and also with those for the $\pm J$ Heisenberg SG under external fields 13.38 .

After establishing the occurrence of a finitetemperature CG transition, we next wish to re-examine via the finite-size scaling analysis the issue whether the standard SG order occurs at the same temperature with the CG order or not. In Fig. 8 we show a finite-size scaling plot of the SG order parameter $q_{\mathrm{SG}}^{(2)}$, assuming a simultaneous $\mathrm{CG}$ and SG transition with a common correlation length exponent, i.e., we set $T_{\mathrm{SG}} / J=0.19$ and $\nu_{\mathrm{SG}}=1.2$. Although the data turn out to scale well for smaller sizes, a significant deviation from the scaling is seen for larger sizes and at lower temperatures. The quality of the scaling is not improved if one tries to adjust $\nu$ to somewhat larger values. A similar poor scaling behavior is also observed even when one instead chooses $\left|1 / T-1 / T_{\mathrm{CG}}\right| T_{\mathrm{CG}} L^{1 / \nu}$ as the scaling variable, and tries to adjust the scaling parameters around $\nu_{\mathrm{SG}}=1.4$. The data for smaller sizes turn out to scale best with choosing $\eta_{\mathrm{SG}}=-0.1$. These parameter values $\nu_{\mathrm{SG}}=1.2$ and $\eta_{\mathrm{SG}}=-0.1$ are close to the values reported by Matsubara et al in Ref. 39. Hence, for the SG order parameter, we have observed a pseudo-critical behavior for smaller sizes, as well as a systematic deviation from the scaling for larger sizes. If the observed deviations were due to the correction-to-scaling, the scaling should be better for larger sizes, which is opposite to our present observation. Therefore, we do not consider the apparent scaling obtained for smaller sizes with $T_{\mathrm{SG}} / J=0.19$ to be acceptable as a true asymptotic scaling.

In Fig. 9 we show a finite-size-scaling plot of the SG order parameter $q_{\mathrm{SG}}^{(2)}$ using the same data as in Fig. 8 but now assuming a zero-temperature $\mathrm{SG}$ transition, i.e., $T_{\mathrm{SG}}=0$ and $\eta_{\mathrm{SG}}=-1$. The value $\eta=-1$ is generically expected for a zero-temperature transition with the nondegenerate ground state. As shown in Fig. 9] the best data collapse is obtained by choosing $\nu_{\mathrm{SG}}=2.2$. If one 


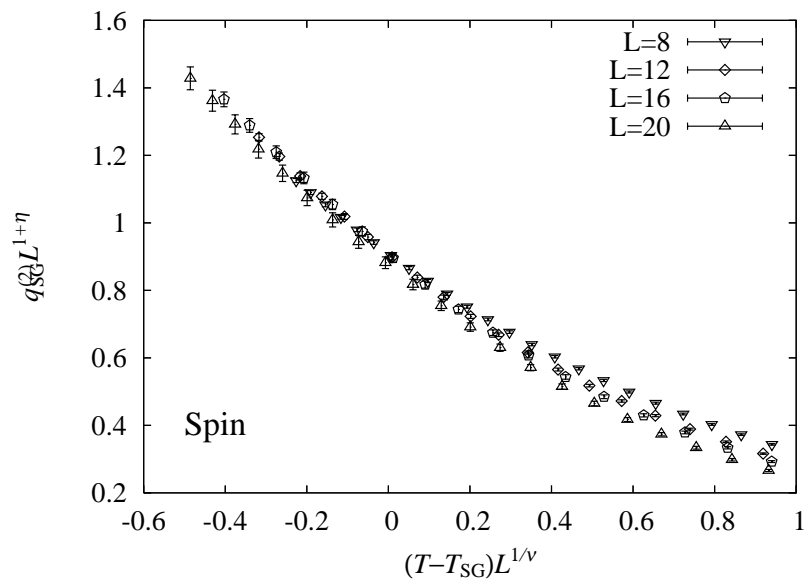

FIG. 8: Finite-size-scaling plot of the squared spin-glass order parameter, assuming a simultaneous chiral-glass and spinglass transition with a common correlation length exponent, i.e., $T_{\mathrm{SG}} / J=0.19$ and $\nu_{\mathrm{SG}}=1.2$. The best scaling is obtained with $\eta_{\mathrm{SG}}=-0.1$.

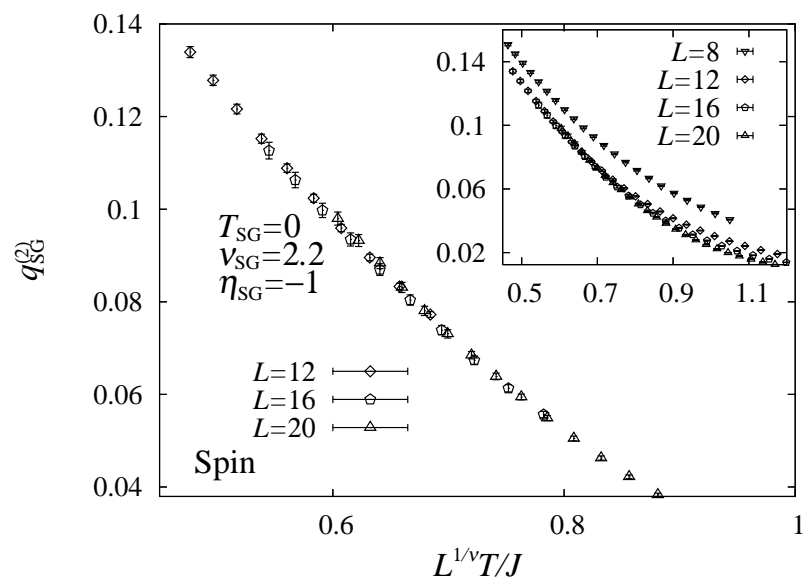

FIG. 9: Finite-size-scaling plot of the squared spin-glass order parameter with assuming a zero-temperature spin-glass transition $T_{\mathrm{SG}}=0$. In the main panel, only the data for larger sizes and at lower temperatures, i.e., those at $T / J \leq 0.19$ and with $L \geq 12$, are plotted. In the inset, the same scaling plot using all the data is shown.

uses in the scaling plot the data at low temperatures, lower than the CG transition temperature $T / J \leq 0.19$, and the data for larger sizes $L \geq 12$, the scaling turns out to work well: See the main panel. By contrast, If one includes in the scaling plot the data for the smallest size $L=8$ and at high temperatures $T / J \geq 0.19$, a significant deviation from the scaling is observed for these data: See the inset. In sharp contrast to the scaling plot of Fig. 8 with $T_{\mathrm{SG}}=T_{\mathrm{CG}}$, we have observed here a better scaling for larger sizes, and a systematic deviation from the scaling for smaller lattices. In that sense, the present finite-size scaling analysis is fully consistent with the occurrence of a $T=0 \mathrm{SG}$ transition, as has long been believed in the community. 4 ,5,6.7.8,9,12,40 Furthermore, the exponent associated with the possible $T=0$ SG transition happens to be rather close to the previous estimates based on the numerical domain-wall method 4.5 .9

Of course, as discussed above, the CG transition occurring at $T / J \simeq 0.19$ would necessarily affect the nature of the SG ordering, even if the Heisenberg spin itself does not order at $T=T_{\mathrm{CG}}$. Thus, even if the SG transition occurs only at $T=0$, an intrinsic SG critical phenomenon associated with this $T=0$ transition should set in at low temperatures below $T_{\mathrm{CG}}$, whereas the data at and above $T_{\mathrm{CG}}$ would be "contaminated" by the CG transition which might well change the ordering behavior of the Heisenberg spin via the associated phase-space narrowing.

Hence, although our present data are fully consistent with the occurrence of the $T=0 \mathrm{SG}$ transition, in order to see such a behavior clearly, one has to choose the scaling region carefully. Inclusion of the data of smaller sizes and at higher temperatures in the analysis would easily deteriorate the quality of the scaling plot, leading to the opposite conclusion. We believe that this is indeed the situation of the recent study of Ref. 39, in which a simultaneous spin and chiral transition at a finite temperature $T_{\mathrm{SG}}=T_{\mathrm{CG}}$ was concluded.

\section{Correlation length}

The temperature dependence of the normalized SG and CG correlation lengths, $\xi_{\mathrm{SG}} / L$ and $\xi_{\mathrm{CG}} / L$, for various sizes are shown in Fig. [10] In contrast to the Binder parameter shown in Fig. [3] the normalized correlation lengths of $L=8$ and 12 shown in in Fig. 10(a) exhibit a clear crossing at a temperature around $T / J \simeq 0.2$ for both cases of the SG and the CG. The observed behavior is consistent with the behavior recently reported by Lee and Young 20 for the $3 D$ Heisenberg SG model with the Gaussian coupling for the sizes up to $L=12$.

We now extend the system size up to $L=20$, and the result is presented in Fig. 10(b). While the crossing temperature for larger sizes $L=16$ and 20 shifts toward lower temperature for both cases of the SG and the $\mathrm{CG}$, the $\mathrm{CG}$ correlation length still has a clear crossing around $T / J \simeq 0.19$, very close to the estimate of $T_{\mathrm{CG}}$ in the previous subsections, with a finite crossing angle. On the other hand, for the SG correlation length, the crossing becomes weaker and almost fades away. Namely, the curves of $L=16$ and 20 merge nearly tangentially with a vanishing crossing angle. The $L=16$ and 20 curves of $\xi_{\mathrm{SG}} / L$ stay on top of each other in an entire temperature region studied below $T / J \simeq 0.19$, as if they were in the critical KT-like phase. Hence, for the SG, with increasing $L$, not simply the crossing temperature shifts toward lower temperature, but the crossing-angle becomes smaller and almost vanishes. This is in contrast to the behavior of the CG correlation length where the 

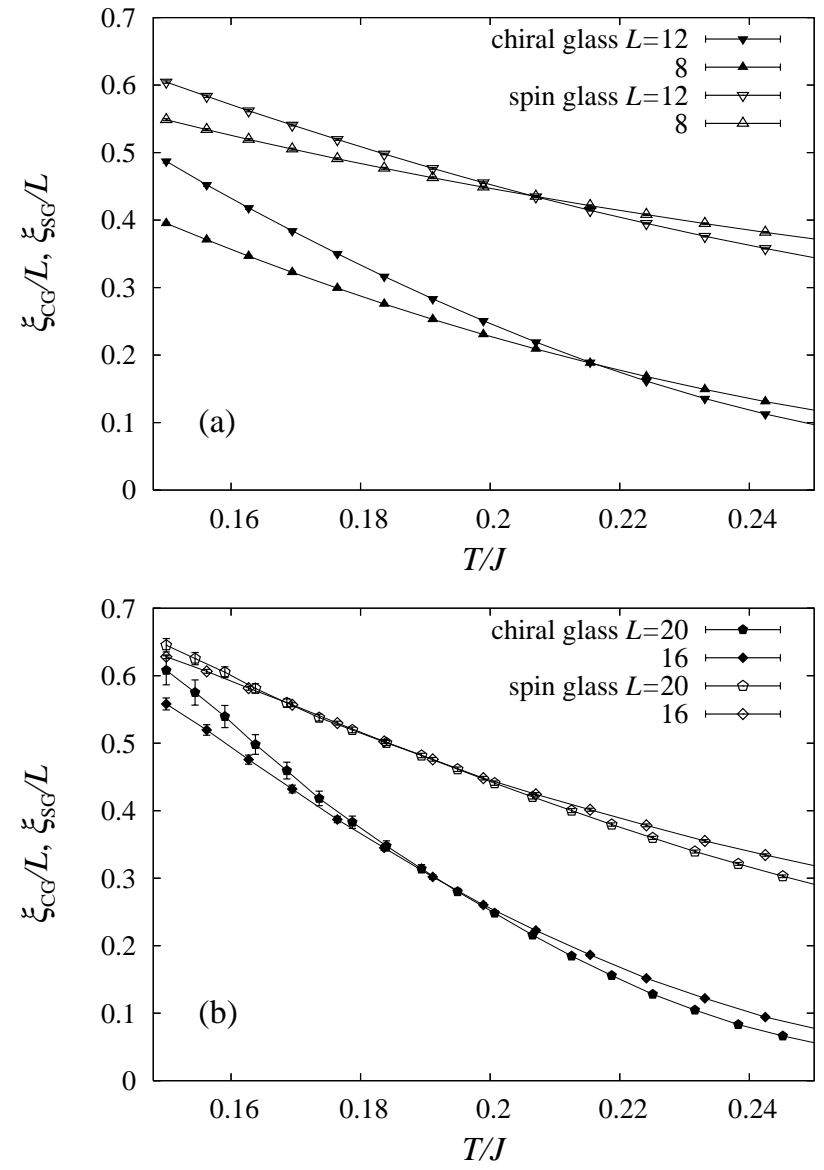

FIG. 10: The correlation length divided by the linear size $L$ plotted against the temperature for the chiral-glass $\xi_{\mathrm{CG}} / L$ (filled symbols) and for the spin-glass $\xi_{\mathrm{SG}} / L$ (open symbols). The data for smaller sizes $(L=8$ and 12) are shown in the upper figure, and those for larger sizes $(L=16$ and 20) are shown in the lower figure.

crossing-angle remains finite with increasing $L$. It thus seems possible that the further increase of $L$ eventually leads to the disappearance of the crossing for $\xi_{\mathrm{SG}} / L$, at least in the temperature range studied here.

This would be consistent with the size-crossover expected from the spin-chirality coupling/decoupling picture, and with our observation in Sec. VB that the decoupling length scale is about $L=20$. Unfortunately, at present, we cannot go to lattices larger than $L=20$ due to the limitation of our computation capability. We certainly expect, however, that the crossing of $\xi_{\mathrm{SG}} / L$ eventually disappears, or at least shifts to a temperature considerably lower than the CG transition temperature $T_{\mathrm{CG}} / J \simeq 0.19$, if we could study lattices considerably larger than $L=20$. For now, we only mention that, although the recent data of the normalized correlation length for smaller lattices of $L \leq 12$ might look rather

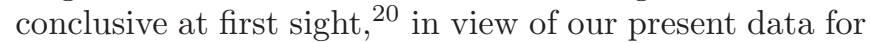
larger sizes presented in Fig. 10 (b), it is still difficult to draw a definite conclusion about the ordering nature of the model only through the correlation-length measurements.

\section{E. Overlap distribution}

In Fig. 11 we show the chiral-overlap distribution function; upper figure (a), and the diagonal-spin-overlap distribution function; lower figure (b), at a temperature $T / J=0.15$ below the $\mathrm{CG}$ transition temperature. One sees from Fig. 11(a) of the chiral-overlap distribution function $P\left(q_{\chi}\right)$ that, with increasing $L$, the side peaks corresponding to the CG EA order parameter $q_{\mathrm{CG}}^{\mathrm{EA}}$ grow and sharpen, which indicates the occurrence of the $\mathrm{CG}$ long-range order. In addition, a central peak at $q_{\chi}=0$ shows up for $L \geq 12$, which also grows and sharpens with increasing $L$. The existence of this central peak coexisting with the side peaks suggests the occurrence of a one-step-like RSB in the CG ordered state. This feature is also consistent with the existence of a negative dip in the CG Binder parameter $g_{\mathrm{CG}}$ and with the crossing of $g_{\mathrm{CG}}$ occurring on the negative side, as was discussed in Sec. $\nabla \mathrm{A}$ The behavior of $P\left(q_{\chi}\right)$ observed here is similar to the previous reports for the 3D Heisenberg SG with the Gaussian coupling 12 and the related Heisenberg SG models ${ }^{13.22 .38}$ By contrast, such a one-step-like feature of the overlap distribution has never seen in the Ising SGs both with the short-range $\frac{41}{2}$ and infinite-range $\frac{42}{2}$ interactions, nor in the Heisenberg SG with the infinite-range interaction 22 .

Fig. 11(b) represents the size dependence of the diagonal-spin-overlap distribution function $P\left(q_{\text {diag }}\right)$ defined by Eq. (18). For larger $L \geq 16$, the distribution function $P\left(q_{\text {diag }}\right)$ has only a single peak at $q_{\text {diag }}=0$, which grows with increasing $L$, without any other divergent peak. This is in sharp contrast to the triple-peak structure observed in the chiral-overlap distribution function $P\left(q_{\chi}\right)$ of Fig. 111 (a), peaked at $q_{\chi}=0$ and $\pm q_{\mathrm{CG}}^{\mathrm{EA}}$. It is also in contrast to the double-peak structure observed in $P\left(q_{\text {diag }}\right)$ of the mean-field Heisenberg SK model, peaked at $q= \pm \frac{1}{3} q_{\mathrm{EA}}{ }^{22}$ As discussed in Sec. IV since the diverging peaks at $q_{\text {diag }}= \pm \frac{1}{3} q_{\text {EA }}$ should arise in $P\left(q_{\text {diag }}\right)$ in the possible SG ordered state with a non-zero EA SG order parameter, ${ }^{22}$ the absence of any divergent peak at non-zero $q_{\text {diag }}$ for larger $L$ strongly suggests that the system is in the SG disordered state even at this low temperature $T / J=0.15$. Interestingly, a closer inspection of Fig. 11 (b) reveals that a weak double-peak structure can be seen for smaller sizes corresponding to the spinchirality coupling regime, $L=8$ and 12 . However, such a double-peak structure in $P\left(q_{\text {diag }}\right)$ tends to disappear for larger sizes corresponding to the spin-chirality decoupling regime, $L \geq 16$. Again, this could be interpreted as the size-crossover from the small-size pseudo SG order to the large-size SG disorder, as is naturally expected from the spin-chirality coupling/decoupling picture. 

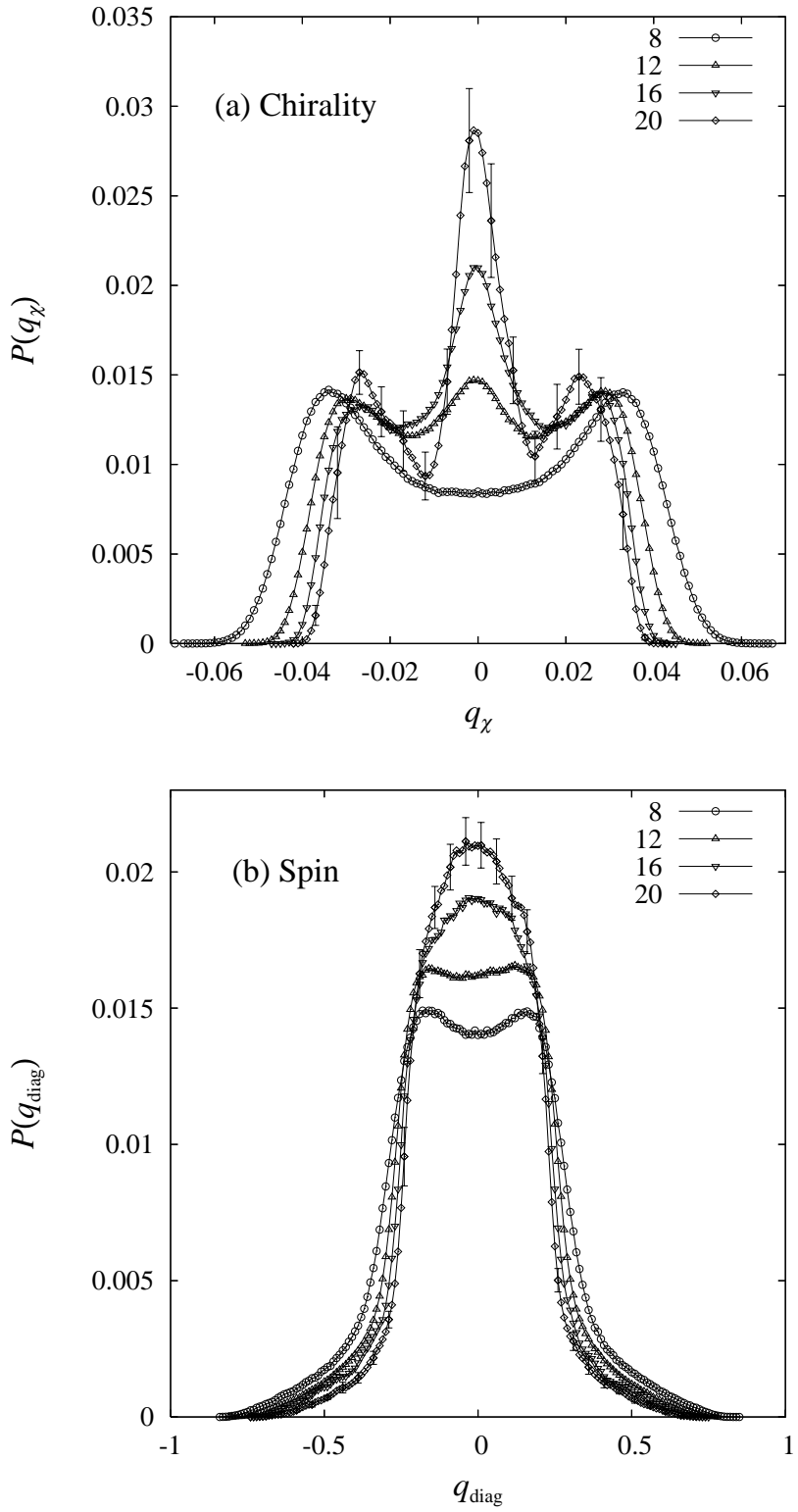

FIG. 11: The size dependence of the chiral-overlap distribution function; upper figure (a), and of the diagonal-spinoverlap distribution function; lower figure (b), at the lowest temperature of the present simulation, $T / J=0.15$.

\section{F. Equilibrium auto-correlation functions}

Next, we discuss the ordering behavior of the model by studying its equilibrium dynamics. In Fig. 12 we show the MC time dependence of the chiral and spin autocorrelation functions for our two largest sizes $L=$ 16 and 20. Here, the time is measured in units of the standard heat-bath MC steps without the temperatureexchange procedure.

In the chiral autocorrelation function $C_{\chi}(t)$ shown in Fig. 12(a), no appreciable difference is observed between the data of $L=16$ and 20 in the time window of $t \leq$
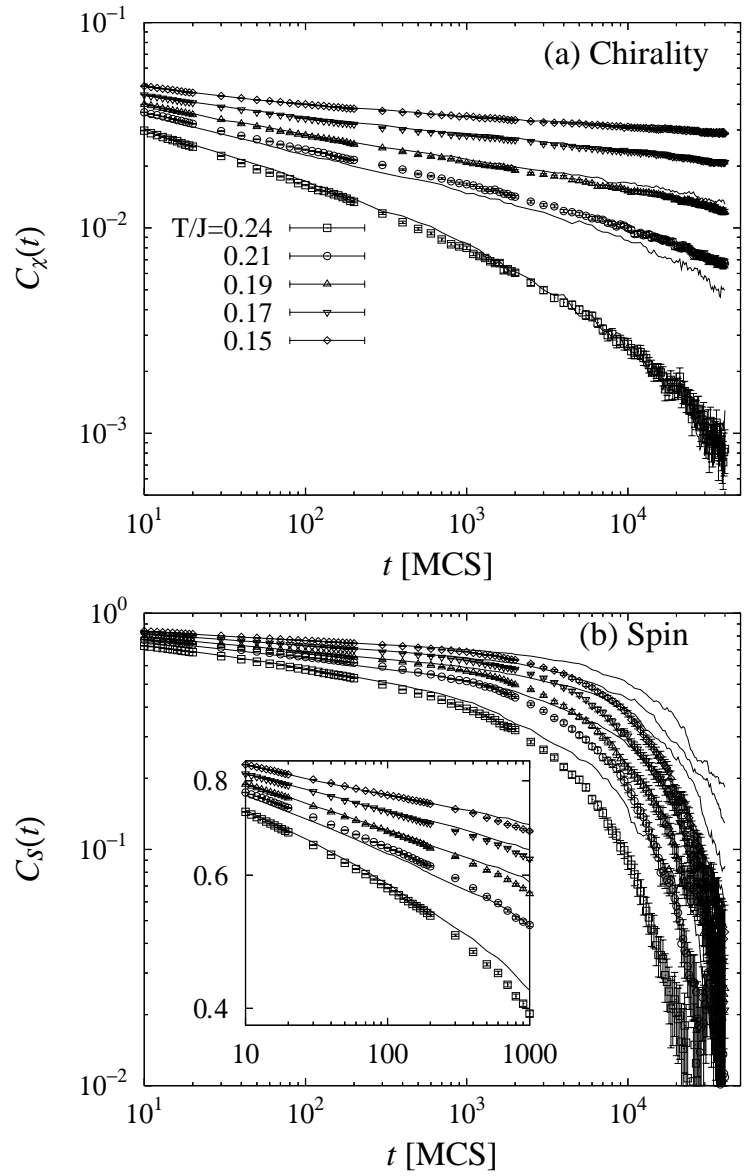

FIG. 12: The Monte Carlo time dependence of the chiral autocorrelation function $C_{\chi}(t)$; upper figure (a), and of the spin autocorrelation function $C_{s}(t)$; lower figure (b), at various temperatures both below and above $T_{\mathrm{CG}} / J \simeq 0.19$. The system size is $L=16$ (given by symbols) and $L=20$ (given by thin lines). The inset is an enlarged view of $C_{s}(t)$ in the short-time region where the finite-size effect is negligible.

$10^{4}$, beyond which a weak size effect is appreciable. The spin autocorrelation function $C_{s}(t)$, by contrast, is more susceptible to the finite-size effect, as can be seen from Fig. [12(b). Even in this case, however, the data in the time window $t \leq 10^{3}$ shows a negligible size effect as shown in the inset.

As can be seen from Fig. 12] a), $C_{\chi}(t)$ shows a downward trend above $T / J=0.19$, an upward trend below $T / J=0.19$, and a near linear behavior at $T / J=0.19$. In order to quantify this, we fit the data at each temperature to the form (27) and plot the $\chi^{2}$-deviation of the fit in Fig. [13 as a function of the temperature around the expected CG transition temperature. The plot has a minimum around $T / J=0.19(1)$, at which the data are optimally fitted to a power law. This estimate of $T_{\mathrm{CG}}$ based on the chiral autocorrelation function agrees well with those obtained from the CG Binder parameter and the $\mathrm{CG}$ order parameter.

We also test a dynamical scaling analysis of the chi- 


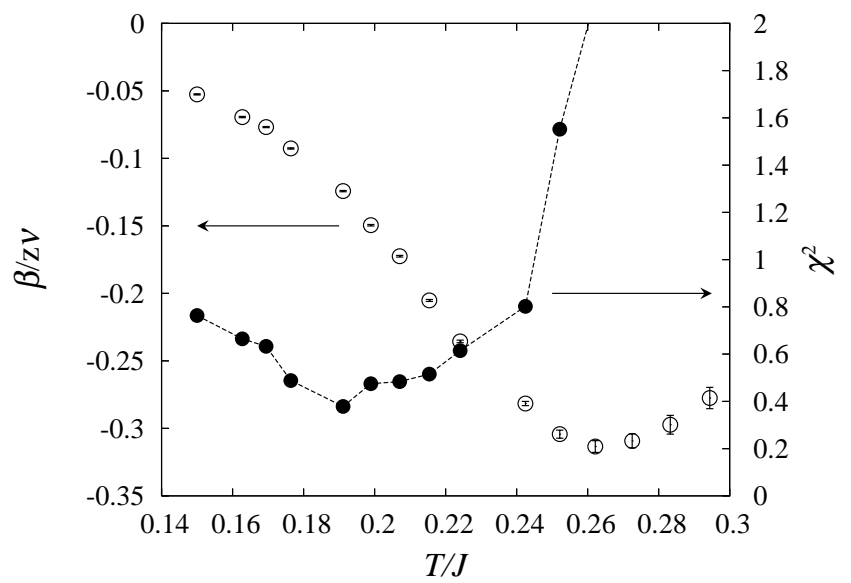

FIG. 13: Residuals per degrees of freedom associated with the $\chi^{2}$-fitting of the chiral autocorrelation function (marked by filled circle), and an estimated effective exponent $\beta / z \nu$ (marked by open circle) plotted against the temperature.

ral autocorrelation function $C_{\chi}(t)$. As can be seen from Fig. 14] the dynamical scaling works well both above and below $T_{\mathrm{CG}}$, with the scaling parameters $T_{\mathrm{CG}} / J=0.195$, $\beta_{\mathrm{CG}}=0.8$ and $z_{\mathrm{CG}} \nu_{\mathrm{CG}}=5.0$. The present estimate of $\beta_{\mathrm{CG}}$ is slightly smaller than, but is not far from the previous estimate of Ref. 40 for the 3D Heisenberg SG with the Gaussian coupling $\beta_{\mathrm{CG}} \simeq 1.1$.

As can be seen from Fig. 12 (b), by contrast, the spin autocorrelation $C_{s}(t)$ shows a downward trend at longer times at any temperature studied, suggesting an exponential-like decay characteristic of the disordered phase. A closer inspection of the data of $C_{s}(t)$ reveals that the data below $T_{\mathrm{CG}}$ exhibit a weak hump-like structure at short times $t \simeq 10^{2}$, though this hump eventually gives way to the down-bending trend characteristic of the disordered phase at longer times $t \gtrsim 10^{3}$ : See the inset of Fig. 12(b). This hump-like structure observed in $C_{s}(t)$ at short times might be a manifestation of the trivial spin-chirality coupling expected at short time scales, and is not likely to be an indication of the SG long-range order, since the downward trend is recovered at longer time scales: See Sec. VI for further details. Hence, from our dynamical data, we conclude again that the CG transition occurs at $T_{\mathrm{CG}} / J=0.19(1)$, without accompanying the simultaneous SG order.

\section{G. Equilibrium correlation time}

Generally, the temporal decay of the autocorrelation is characterized by the temperature-dependent characteristic time scale, the correlation time $\tau(T)$, which represents a dynamical crossover from the short-time critical behavior to the long-time relaxation. The correlation time $\tau(T)$ diverges as $\tau(T) \simeq\left|T-T_{\mathrm{c}}\right|^{-z \nu}$ when the temperature approaches $T_{\mathrm{c}}$ from above. One promising method of estimating $\tau$ from the autocorrelation function has been

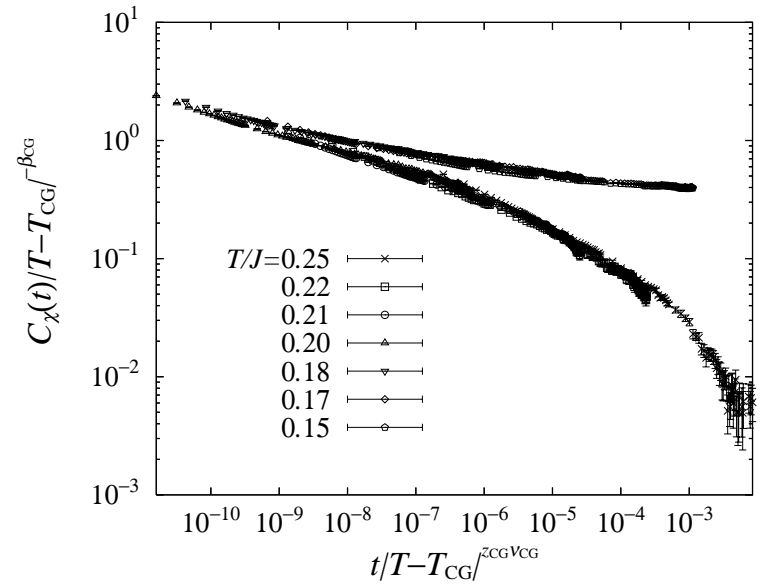

FIG. 14: Dynamical scaling plot of the chiral autocorrelation function. The best scaling is obtained with choosing $T_{\mathrm{CG}}=0.19, \beta_{\mathrm{CG}}=0.8$ and $z_{\mathrm{CG}} \nu_{\mathrm{CG}}=5.4$. The upper branch represents the scaling of the data below $T_{\mathrm{CG}}$, while the lower branch represents that above $T_{\mathrm{CG}}$.
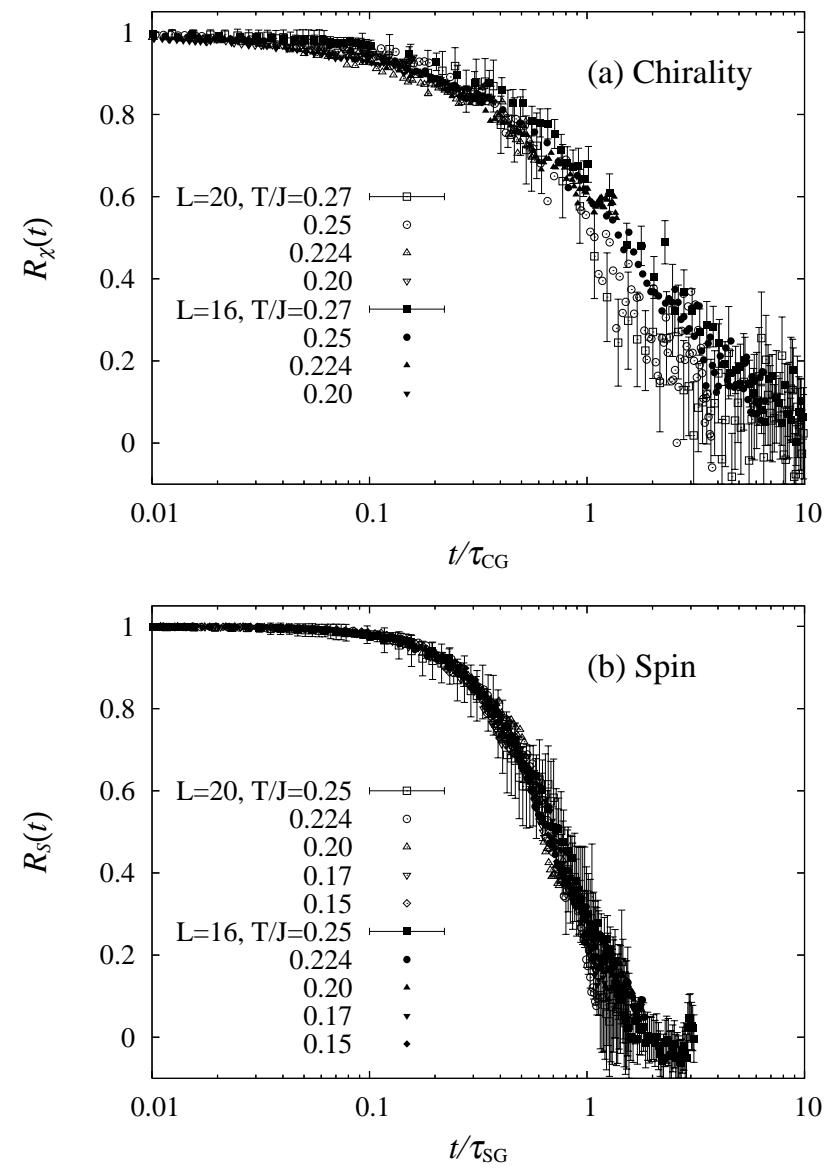

FIG. 15: Scaling plot of the chiral ratio function; upper figure (a), and of the spin ratio function; lower figure (b), at various temperatures for the sizes $L=16$ and 20 . 
proposed by Bhatt and Young 43 , who employed a scaling analysis of the dynamical ratio function. For the spin autocorrelation, this reads as

$$
R_{s}(t)=\frac{C_{s}(t)}{\sqrt{\frac{1}{N}\left[\left\langle\left(\sum_{i} \vec{S}_{i}\left(t_{0}\right) \cdot \vec{S}_{i}\left(t+t_{0}\right)\right)^{2}\right\rangle\right]}} .
$$

The corresponding chiral ratio function is defined by

$$
R_{\chi}(t)=\frac{C_{\chi}(t)}{\sqrt{\left[\left\langle\left(\frac{1}{3 N} \sum_{i \mu} \chi_{i \mu}\left(t_{0}\right) \chi_{i \mu}\left(t+t_{0}\right)\right)^{2}\right\rangle\right]}} .
$$

Because the ratio function is dimensionless, the prefactor $t^{-\beta / z \nu}$ in Eq. (28) is canceled out. The dynamical scaling form of $R(t)$ is then given as a single-variable function of $t / \tau$

$$
R(t)=\bar{R}(t / \tau)
$$

where $\bar{R}$ is a scaling function. If one appropriately chooses the scaling parameter $\tau$ which depends on the temperature and the system size, the ratio functions should be scaled on to a single curve. Using this method, Bhatt and Young ${ }^{43}$ successfully estimated the correlation time of the short-range Ising EA model and the meanfield Ising SK model. Subsequently, this method has been extended to non-equilibrium relaxation, where the ratio function depends not only on the measurement time $t$ but also on the waiting time $t_{w}$. The off-equilibrium method was applied recently by Matsumoto, Hukushima and Takayama to the $3 D \pm J$ Heisenberg $\mathrm{SG}^{44}$

Here we use this method to estimate the correlation times both for the spin and for the chirality. In comparison with the previous off-equilibrium study, ${ }^{44}$ the present equilibrium study has an advantage that one needs not extrapolate to an equilibrium limit, i.e., needs not take the $t_{w} \rightarrow \infty$ limit. In Fig. 15 we show the scaling plot of the chiral and spin ratio functions. We note that both the spin and chiral scaling functions are described roughly by an exponential form.

In order to compare the spin and chiral correlation times, denoted by $\tau_{\mathrm{SG}}$ and $\tau_{\mathrm{CG}}$, respectively, we plot them in Fig. 16] as a function of the temperature. In the figure, we combine the data with those obtained in a wider temperature range by off-equilibrium simulation of Ref. 44. As can be seen from Fig. 16] the chiral correlation time is shorter than the spin correlation time at higher temperatures, similarly to the behavior of the correlation length discussed in Sec. VD As the temperature is decreased, the chiral correlation time $\tau_{\mathrm{CG}}$ grows faster than the spin correlation time $\tau_{\mathrm{SG}}$, and eventually exceeds $\tau_{\mathrm{SG}}$ at a certain characteristic temperature $T=T_{\times}(L)$. The size dependence of this crossover temperature $T=T_{\times}(L)$ is apparently weak: We get $T=T_{\times} \simeq 0.24$ both for $L=16$ and 20 . It strongly suggests that, even in the thermodynamic limit,

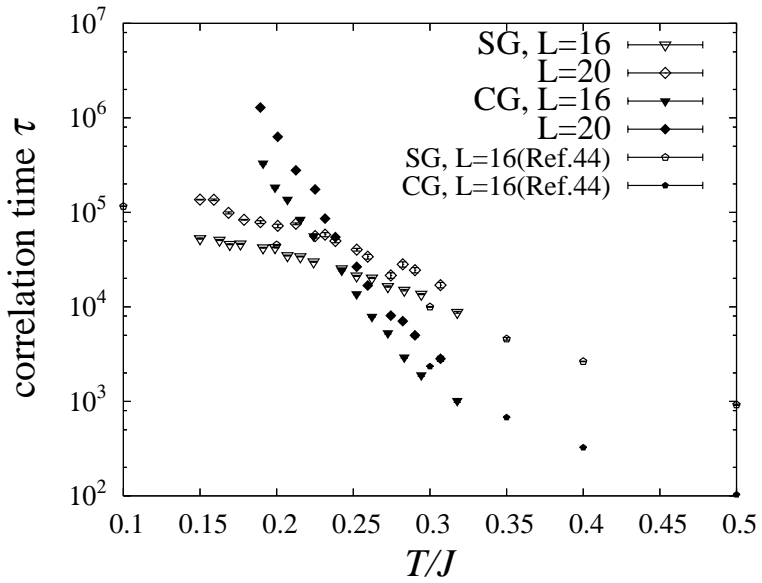

FIG. 16: The temperature dependence of the the chiral and spin correlation times for the sizes $L=16$ and 20. The corresponding data obtained from the off-equilibrium simulation of Ref. 44 are also included.

the chiral correlation time exceeds the spin correlation time at a crossover temperature $T_{\times}(\infty)$, which is located somewhat above the CG transition temperature $T_{\mathrm{CG}} / J \simeq 0.19$. It means that, with decreasing the temperature, the relevant degree of freedom dominating the long-time ordering behavior changes from the spin to the chirality at $T=T_{\times}$. In order to further illustrate this changeover, we show in Fig. 17 the time-dependence of the spin and chiral ratio functions at two representative temperatures $T / J=0.25$ and 0.20 , each above and below $T_{\times}$. As can be clearly seen from the figure, with decreasing the temperature across $T_{\times}$, the temporal decay of the chiral ratio function becomes much slower than that of the spin ratio function: Compare the two arrows in the figure.

The time scale associated with such a crossover, $t_{\times}$, is roughly estimated to be $10^{5} \sim 10^{6} \mathrm{MCS}$. For more precise estimate of $t_{\times}$, more quantitative analysis of the size dependence of the crossover time scale would be necessary. Naturally, this crossover time $t_{\times}$gives a measure of the time scale above which the spin-chirality decoupling can be observed in dynamics. Thus, the spin-chirality decoupling in the dynamics would be eminent only at temperatures lower than $T_{\times} / J \simeq 0.24$ and at times longer than $t_{\times} \simeq 10^{5}-10^{6}$ MCS. This crossover time scale is rather long, yet, is finite. It is important to realize that, in order to resolve the controversy concerning the presence or the absence of the spin-chirality decoupling in the Heisenberg $\mathrm{SG}$, one has to probe the equilibrium dynamics beyond this crossover time scale $10^{5}-10^{6}$ MCS at temperatures lower than $T_{\times} / J \simeq 0.24$, about some $20 \sim 30 \%$ above $T_{\mathrm{CG}} \stackrel{45}{\underline{4}}$

As argued in Sec. III from the spin-chirality coupling/decoupling picture, a similar phenomenon is expected also in the spatial correlation of the model in terms of the length scale. Namely, one expects that at a certain crossover temperature $T_{\times}^{\prime}$, which is prob- 


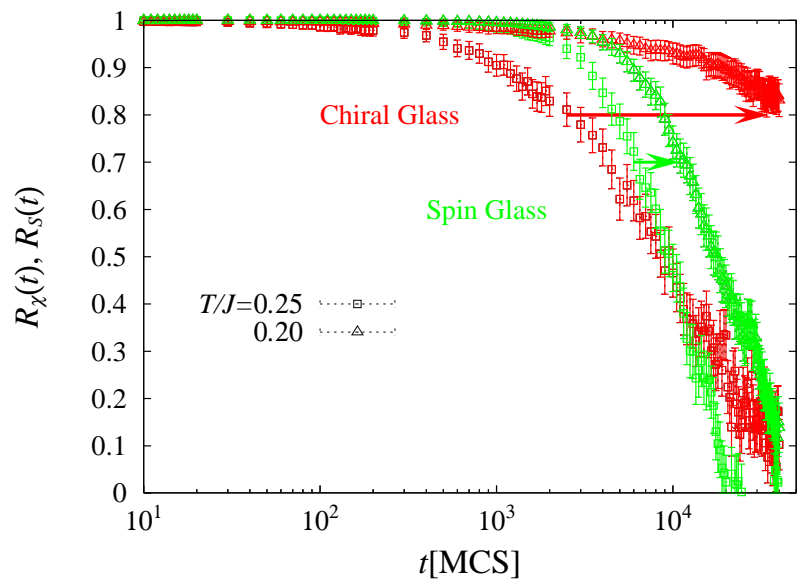

FIG. 17: Temporal decay of the chiral and spin ratio functions at temperatures $T / J=0.25$ and 0.20 . The system size is $L=16$. With decreasing the temperature from $T / J=0.25$ to 0.20 , the chiral relaxation slows down much more slowly than the spin relaxation, as illustrated by the arrows.

ably close to the dynamical crossover temperature $T_{\times}$ discussed above, the CG correlation length $\xi_{\mathrm{CG}}$ exceeds the SG correlation length $\xi_{\mathrm{SG}}$. This changeover of the dominant length scale gives a crossover length scale $L_{\times}$ above which the spin-chirality decoupling is eminent in spatial correlations. Unfortunately, unlike the case of the correlation time, the limitation of the available system size prevents us from directly estimating $L_{\times}$. In Fig. 18] we plot the temperature dependence of the CG and SG correlation lengths for the sizes $L=16$ and 20 . For these sizes, the crossing of $\xi_{\mathrm{SG}}$ and $\xi_{\mathrm{CG}}$ occurs at a temperature lower than the CG transition temperature, in contrast to the case of the correlation time. Nevertheless, the crossover temperature at which $\xi_{\mathrm{SG}}$ and $\xi_{\mathrm{CG}}$ of finite $L$ cross, tends to increase with increasing $L$. If we roughly estimate the crossover length scale of finite systems by extrapolating the data of Fig. 18 we tentatively get $L_{\times} \simeq 11(L=16)$ and $L_{\times} \simeq 14(L=20)$. These results are certainly not inconsistent with our estimate of $L_{\times} \simeq 20$ based on the behaviors of the SG order parameter, the dimensionless correlation length $\xi / L$ and other quantities.

\section{H. The $A$ and $G$ parameters}

We have also calculated the $A$ and $G$ parameters defined in Sec. IV both for the CG and SG orders. In Fig. 19] the temperature and size dependence of the $A$ and $G$ parameters for th $\mathrm{CG}$ order, $A_{\mathrm{CG}}$ and $G_{\mathrm{CG}}$, is shown. Although the data are rather noisy due to the large sample-to-sample fluctuations, the $A$ parameter of different $L$ show a crossing and a peak around the expected CG transition point $T / J \simeq 0.19$, as can be seen from Fig. 19] a). In particular, with increasing $L, A_{\mathrm{CG}}$ stays non-zero below $T_{\mathrm{CG}}$, indicating that the $\mathrm{CG}$ or-

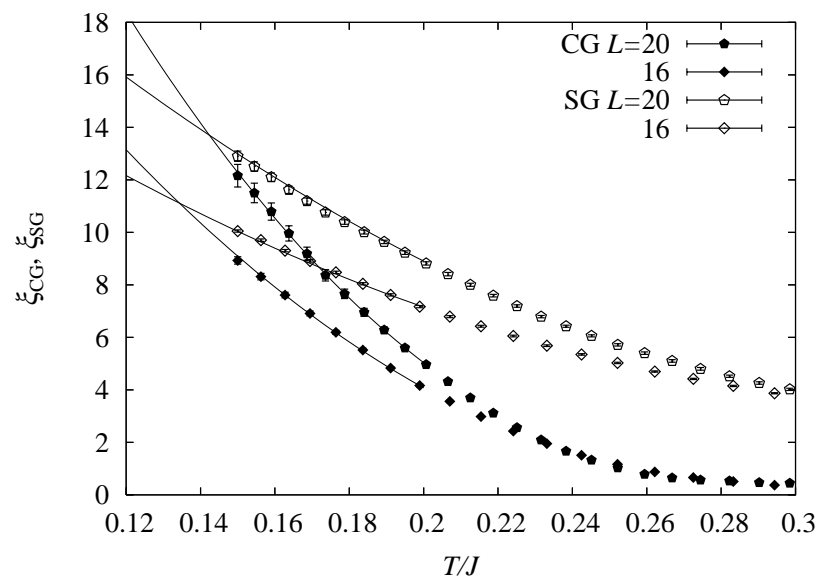

FIG. 18: The temperature dependence of the chiral and spin correlation lengths for finite systems. The system size is $L=$ 16 and 20. The data are the same as those shown in Fig. 10] but not divided by $L$ here. The curves are polynomial fits of the data which are extrapolated to lower temperatures to deduce the crossing temperature given in the text.
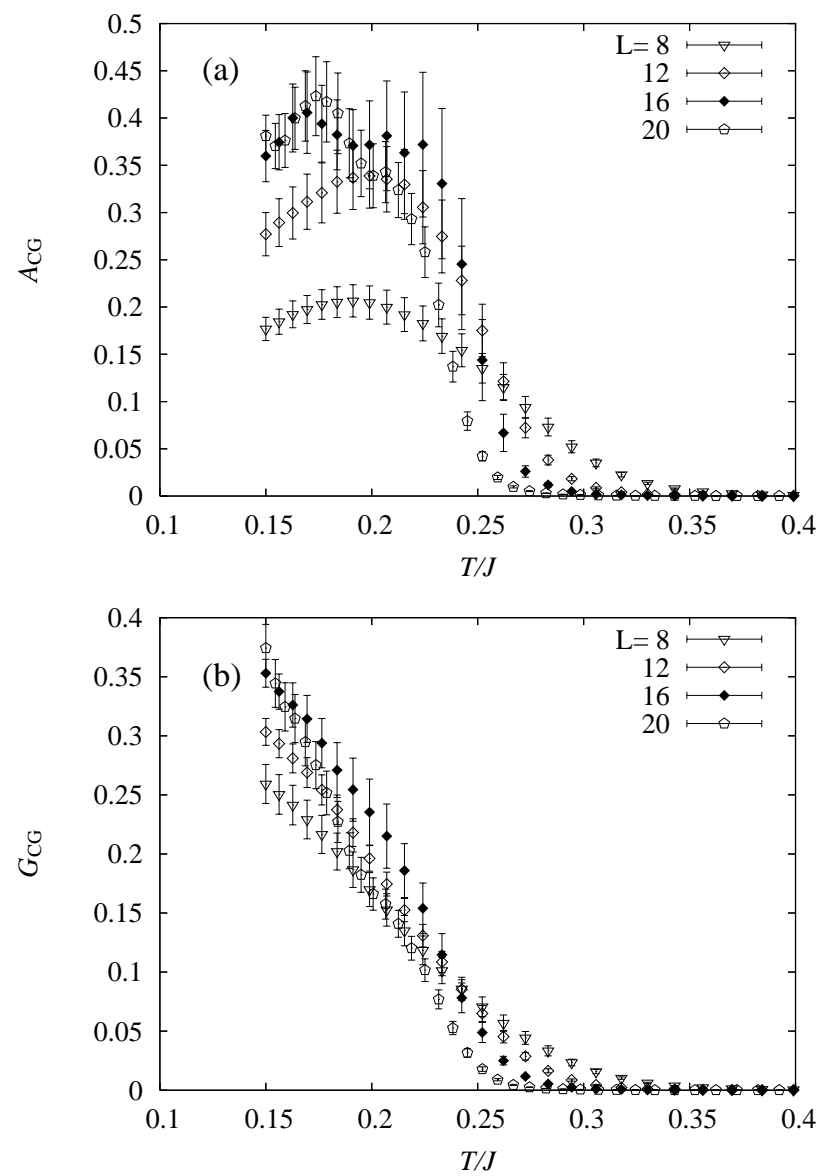

FIG. 19: The temperature and size dependence of the $A$ parameter of the chirality; upper figure (a), and of the $G$ parameter of the chirality; lower figure (b). 

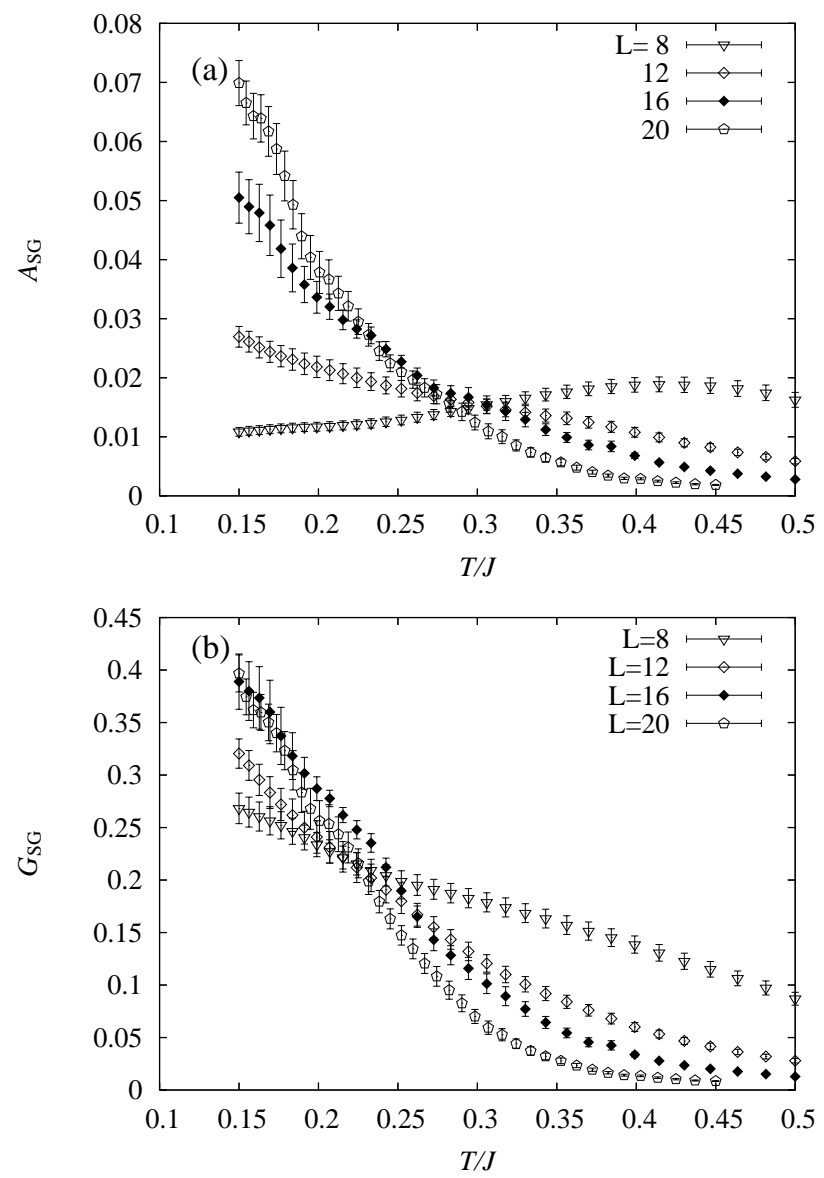

FIG. 20: The temperature and size dependence of the $A$ parameter of the spin; upper figure (a), and of the $G$ parameter of the spin; lower figure (b).

dered state is non-self-averaging. These findings combined with the peculiar shape of $P\left(q_{\chi}\right)$ shown in Sec. $\mathrm{\nabla E}$ suggest that the CG ordered phase accompanies an RSB with a non-self-averageness. For the corresponding $G$ parameter, the crossing is not so clear, as is shown in Fig. 191(b).

The temperature and size dependence of the $A$ and $G$ parameters for th $\mathrm{SG}$ order, $A_{\mathrm{SG}}$ and $G_{\mathrm{SG}}$, is shown in Fig. 20] As shown in Fig. [20] a), the $A$ parameter exhibits a crossing, although the crossing temperature is located considerably above $T_{c}$ for this range of $L$. One might be tempted to interpret such a crossing of $A_{\mathrm{SG}}$ as an unambiguous evidence of the occurrence of the standard SG transition. However, one has to be careful here: Although the crossing of $A_{\mathrm{SG}}$ is certainly a signature of some sort of phase transition occurring there, it does not necessarily mean the occurrence of the standard SG transition characterized by a non-zero SG order parameter. A non-zero $A_{\mathrm{SG}}$ persisting in the $L \rightarrow \infty$ limit simply means that the SG order parameter $q_{\mathrm{SG}}^{(2)}$, or the SG susceptibility $\chi_{\mathrm{SG}}=N q_{\mathrm{SG}}^{(2)}$, is non-self-averaging. Below the $\mathrm{CG}$ transition temperature, one expects that the $\mathrm{SG}$ order parameter is still Gaussian-distributed around zero with a width corresponding to the finite SG susceptibility $\chi_{\mathrm{SG}}$, while the width exhibits sample-to-sample fluctuations leading to the non-self-averaging $\chi_{\mathrm{SG}}$. The latter is a natural consequence of the phase-space narrowing which should inevitably accompany the CG transition with the one-step-like RSB. Hence, the crossing of $A_{\mathrm{SG}}$, and a finite $A_{\mathrm{SG}}$ remaining in the $L \rightarrow \infty$ limit below $T_{\mathrm{CG}}$, are compatible with the absence of the standard SG long-range order, and is entirely consistent with the the CG transition not accompanying the standard SG long-range order.

As shown in Fig. 20 b), the $G$ parameter of the spin exhibits a crossing around $T_{\mathrm{CG}}$. The relation Eq. (24), combined with our observation in Fig. B(b), indicates that $G_{\mathrm{SG}}$ also takes a non-zero value below $T_{\mathrm{CG}}$. Thus, the observed crossing of $G_{\mathrm{SG}}$ is just as one expects for the CG transition. In other words, one cannot interpret the crossing of $G_{\mathrm{SG}}$ as an indicator of the onset of the standard SG long-range order.

\section{DISCUSSIONS}

In this section, in view of our MC results presented in the previous section, we wish to examine and discuss the recent numerical studies on the 3D Heisenberg SG. Many of these studies suggested, contrary to our present study, that the spin and the chirality ordered simultaneously at a finite temperature with a common correlation length exponent $\nu_{\mathrm{SG}}=\nu_{\mathrm{CG}}$, i.e., no spin-chirality decoupling in the 3D Heisenberg SG 16.18.19.20.39.46 Below, we wish to make some comments on these numerical works from the standpoint of the spin-chirality coupling/decoupling picture.

\section{A. Stiffness method}

First, we wish to discuss the analyses based on the stiffness method, $\stackrel{16,17.47}{1 n}$ this method, one computes by some numerical means the change of the ground-state energy of finite systems of size $L$ under the appropriate change of boundary conditions imposed on the system. This energy is called a stiffness energy (or a domain-wall energy), $\Delta E_{L}$, which gives a measure of an energy scale of low-energy excitations of size $L$. For large $L, \Delta E_{L}$ is expected to behave as a power-law, $\Delta E_{L} \approx L^{\theta}, \theta$ being a stiffness exponent. If $\theta<0$, the system remains in the disordered state at any nonzero temperature, whereas if $\theta>0$ the system possesses a finite long-range order at low enough temperatures with $T_{c}>0$. Here, we discuss this method first in conjunction with the detection of the standard SG order, leaving the detection of the CG order later.

The nontrivial part of this stiffness method concerns with the choice of the boundary conditions employed in computing the stiffness energy. There could be various 
choices, and the behavior of $\Delta E_{L}$ might in principle depend on these choices particularly for small sizes accessible in numerical simulations. The most standard choice is the combination of the periodic and the antiperiodic boundary conditions (P/AP). In the case of the Heisenberg SG, the $\mathrm{P} / \mathrm{AP}$ combination necessarily accompanies a flipping of the chirality (remember that the chirality of the Heisenberg spin is odd under the spin inversion $\vec{S} \rightarrow-\vec{S}$ ), so that the $\mathrm{P} / \mathrm{AP}$ combination should detect the chiral order for large enough $L \underline{\underline{9}}$ In order to detect the standard SG order independently of the CG order by this stiffness method, Ref. 9 introduced the "rotation" boundary conditions (ROT), which imposed a $\pi$ rotation on the boundary spins without flipping the chirality, which was combined with the standard $\mathrm{P}$ boundary conditions in calculating the stiffness energy $\frac{9}{}$ Such a P/ROT combination applied to the 3D Heisenberg SG yielded a negative $\theta$, i.e., $\theta \sim-0.51$ for the Gaussian coupling, and $\theta \sim-0.49$ for the $\pm J$ coupling, which implied the absence of the standard SG order at nonzero temperature ${ }^{9}$

By contrast, Matsubara, Endoh and Shirakura proposed a different choice of boundary conditions in computing $\Delta E_{L}$, i.e., to use the free (open) boundary conditions as a reference and impose the rotational-twist to such "optimized" spin configurations obtained under the free boundary conditions in which the stress at the boundary is released ${ }^{16.17}$ These authors observed that the stiffness exponent evaluated in this way was largely positive, close to the spin-wave exponent $\theta=1$, and argued that the 3D Heisenberg SG exhibited a finitetemperature SG transition. The method similar in spirit to the one used in Refs. 16 and 17 was also applied to the $X Y$ SG by Kosterlitz and Akino $\stackrel{47}{\longleftarrow}$ leading to the similar conclusion. Thus, the result obtained by applying the free/twisted-free $(\mathrm{F} / \mathrm{TF})$ boundary conditions, $\theta>0 \mathrm{im}-$ plying $T_{\mathrm{SG}}>0$, is in sharp contrast to the result obtained by applying the $\mathrm{P} / \mathrm{ROT}$ boundary conditions, $\theta<0 \mathrm{im}-$ plying $T_{\mathrm{SG}}=0$. Discrepancy between the stiffness exponents evaluated by the different choices of boundary conditions was also observed in other SG models, e.g., in the $2 \mathrm{D}$ Ising $\mathrm{SG}, 48$

Although the authors of Refs. 16 and 17 argued that their "optimized" boundary conditions were superior to the other choices, a theoretical basis of such a claim seems not so obvious. For example, one might make the following counter-argument that one should not optimize the boundary conditions in calculating $\Delta E_{L}$ : In the spirit of the domain-wall renormalization-group idea by Bray and Moore $\stackrel{49}{\underline{9}}, \Delta E(L)$ represents an energy scale associated with the interaction between the coarse-grained blocks of size $L$ in an infinite SG sample. Since these blocks are necessarily subject to the strong frustration effect caused by the interaction with the neighboring blocks surrounding them, an optimization of their energy, independently at each block ignoring the inevitable frustration effect due to the interaction with the neighboring blocks, is hardly compatible with the original RG idea. One may thus argue that, in calculating $\Delta E_{L}$ in SGs, one should not make the optimization of boundary conditions referring to the particular bond realization of each sample.

Concerning the apparent difference of the stiffness exponents arising from the different choices of boundary conditions, there generally exist two possibilities: Either, (i) the observed difference is a finite-size effect where there is a single stiffness exponent for large enough lattices independent of applied boundary conditions, or, (ii) the observed difference is a bulk effect which persists even in large enough lattices. In the case of the 2D Ising SG, Carter, Bray and Moore numerically observed that, although both the $\mathrm{P} / \mathrm{AP}$ and $\mathrm{F} / \mathrm{TF}$ boundary conditions yielded the same stiffness exponent asymptotically for large $L$, i.e., the possibility (i) above, the finite-size effect was much reduced in the $\mathrm{P} / \mathrm{AP}$ than in the $\mathrm{F} / \mathrm{TF}, 48$ For vector SG, there so far exists no convincing evidence which of the above (i) and (ii) is really the case. In any case, a practical question we face with is which set of boundary conditions gives a true asymptotic answer from smaller sizes accessible in simulations.

One plausible criterion might be that, among all possible excitations in the system, the one giving the lowest excitation energy $\Delta E_{L}$, or equivalently, the one giving the smallest stiffness exponent $\theta$, should be chosen. The reason is simply because among all possible excitations the one with the lowest excitation energy should be most efficient in destroying the order so long as it has non-negligible weight in the thermodynamics, and would dominate the low-energy dynamics of the model. Under this criterion, when the different choices of boundary conditions yield different $\theta$ values, the one giving the smallest $\theta$, or the most negative $\theta$, should be chosen. In particular, when one set of boundary conditions yields a positive $\theta$ while the other yields a negative $\theta$, the one giving a negative $\theta$ should be chosen. If so, in the case of the 3D Heisenberg SG of our interest, the P/ROT combination without any optimization procedure should be chosen since it gives the lowest $\theta$ (negative $\theta$ ) reported so far ${ }^{9}$ This suggests that the standard SG order in the 3D Heisenberg SG occurs only at $T=0$. Of course, it is still possible that some other type of boundary conditions might yield a still smaller $\theta$, but it does not change the conclusion that the SG order occurs only at $T=0$.

It should also be remembered that the types of lowenergy excitations generated via a particular choice of boundary conditions is only a subset of all possible excitations in the system: They are basically wall-like excitations, not including more complex excitations like, say, a "vortex" excitation which is possible in the Heisenberg SG reflecting the $S O(3)$ nature of its order parameter space, 50 or a "sponge" excitation which is closely related to the RSB structure of the ordered state 51.52 Unfortunately, we have little knowledge concerning what is the most relevant low-energy excitation governing the ordering of the system, and hence, have no well-based criterion to choose one set of boundary conditions from the others as superior. Although we feel that our argument above 
speaks for a zero-temperature SG transition in the 3D Heisenberg SG, it would be fair to say at present that no definitive conclusion can be drawn solely based on this stiffness method.

We finally wish to refer to the stiffness method in detecting the chiral order. As mentioned, since the sign of the chirality is flipped by changing the boundary conditions from $\mathrm{P}$ to $\mathrm{AP}$, the most standard $\mathrm{P} / \mathrm{AP}$ combination could be used in detecting the chiral order, at least for large enough $L$. In practice, however, the application of the reflecting $(\mathrm{R})$ boundary is more efficient in detecting the chiral order, as shown in Ref. 9. The chiral stiffness exponent of the 3D Heisenberg SG determined in this way turned out to be positive implying a CG transition occurring at a nonzero temperature ${ }^{9}$ Other authors also reported a positive value for the chiral stiffness exponent both for the 3D $X Y \mathrm{SG}^{47}$ and the 3D Heisenberg $\mathrm{SG}^{17}$.

\section{B. Equilibrium dynamics}

Matsubara, Shirakura and Endoh reported a further evidence of the simultaneous spin and chiral transition in the 3D Heisenberg SG by investigating the equilibrium spin dynamics 18 . In order to eliminate the effect of global spin rotations inherent to finite systems, Matsubara et al introduced an artificial global-rotation correction in the spin dynamics of the model. They observed that the modified spin autocorrelations adjusted by the globalrotation correction exhibited at lower temperatures a tendency to approach a nonzero value at longer times, which was interpreted as an evidence of a finite SG long-range order.

It should be noticed here that, when one looks at a quantity which is even under the symmetry transformation of the Hamiltonian like the modified spin autocorrelation function of Ref. 18, one needs to examine its size dependence carefully. As is well-known, an even quantity in finite systems always takes a nonzero value even above $T_{c}$ due to the finite-size effect, where this nonzero value decreases with the size $L$, eventually vanishing as $L \rightarrow \infty$ above $T_{c}$. (Indeed, in an extreme occasion of a single spin, the modified spin autocorrelation function as computed by Matsubara et al does not decay at all even at an infinite temperature!) The ordering behavior of the modified spin autocorrelation as observed by Matsubara et al might possibly be caused by the finite-size effect. In order to refute such suspicion, one needs to study its size dependence carefully, whereas the analysis of Ref. 18 was limited to a fixed size $L=16$.

It should be stressed that, even within the spinchirality coupling/decoupling scheme, it is still possible that the spin autocorrelation function $C_{s}(t)$ of an infinite system exhibits below $T_{\mathrm{CG}}$ a hump-like weak structure at short times as illustrated in Fig[2] which is an echo of the plateau-like structure of the chiral autocorrelation function. In the temperature range $T_{\mathrm{SG}}<T<T_{\mathrm{CG}}$, such a hump of $C_{s}(t)$ appears only at times shorter than the crossover time scale $t_{\times}\left(t_{\times}\right.$was estimated to be $t_{\times} \approx 10^{5}-10^{6}$ above $T_{\mathrm{CG}}$ ), while $C_{s}(t)$ eventually decays at long enough times $t>t_{\times}$Indeed, as was shown in the inset of Fig. 12 such a hump-like weak structure of the spin autocorrelation was discernible in our present data of $C_{s}(t)$ at short times $t \simeq 10^{2}$, which, however, eventually decayed toward zero at longer times.

Berthier and Young also observed in their recent offequilibrium simulation of the 3D Heisenberg SG a weak hump-like structure in the spin autocorrelation in the time range $t \lesssim 10^{4}$, which corresponded to the quasiequilibrium regime ${ }^{46}$. These authors interpreted the observed hump as an evidence of a nonzero SG long-range order at that temperature. As noted above, however, such a hump is also consistent with the the spin-chirality coupling/decoupling picture as long as the hump is observed only at shorter times $t<t_{\times}$.

\section{Nonequilibrium dynamics}

Nakamura and Endoh applied a non-equilibrium method to study the SG and the CG orderings of the 3D $\pm J$ Heisenberg SG $\stackrel{19}{=}$ Analyzing the time dependence of the initial growth of the SG and the CG susceptibilities with use of a dynamical scaling, these authors concluded that the spin and the chirality ordered simultaneously at a finite temperature $T / J=0.21 \sim 0.22$. While the lattice size studied $L \leq 59$ was rather large, the crucial question to be addressed is whether the long-time limit $t \rightarrow \infty$ was safely taken justifying the use of the dynamical scaling. In other words, although the nominal lattice size studied was large, the equilibrated length scale actually probed in these off-equilibrium simulations might be rather short. In fact, their non-equilibrium method is uncontrolled with respect to the time scale toward equilibrium. Since the equilibration time could easily become a huge number in SG, care has to be taken as regards the equilibrated length scale actually probed by this type of non-equilibrium simulation. As one judges from the maximum values of the SG and the CG susceptibilities reached by the off-equilibrium simulation of Ref. 19, the "dynamical correlation length" still remained rather short: Namely, even around the transition temperature $T_{\mathrm{CG}} / J \simeq 0.2$, it reached around 10 lattice spacings for the spin, and only 1 or 2 lattice spacings for the chirality. The dynamical chiral correlation length stayed particularly short. This is consistent with a recent off-equilibrium simulation by Berthier and Young in which the dynamical chiral correlation length stayed much shorter than the dynamical spin correlation length in the investigated time range ${ }^{46}$. Here note that, irrespective of the question of whether the CG transition accompanies the simultaneous SG transition or not, the chiral correlation length in equilibrium should diverge at and below the $\mathrm{CG}$ transition temperature $T_{\mathrm{CG}} / J \simeq 0.2$. Hence, the observation above simply tells that, even at 
the maximum simulation time of Refs 19 and 46 , the system still stayed in an extreme initial time regime. In order to deduce the equilibrium ordering properties from these off-equilibrium data, one is forced to extrapolate the behavior around $\xi_{\mathrm{CG}} \sim 1$ to $\xi_{\mathrm{CG}}=\infty$, which could be dangerous in the present model since the model might possess the characteristic crossover length scale at around 20 .

One may feel that the dynamical spin correlation length reached in the off-equilibrium simulation of Ref. $19, \xi \approx 10$, might be reasonably large for deducing the ordering properties of the spin. However, we feel it is not enough. This length scale of 10 is still not large enough compared with the crossover length scale estimated in the present work $L_{\times} \approx 20$. Remember that the spin-chirality decoupling, if any, is a long scale phenomena observable at length scale longer than $L_{\times}$. Second, in the off-equilibrium simulations of Refs. 19 and 46, even when the dynamical SG correlation length grows around 10 lattice spacings, the $Z_{2}$ chiral degree of freedom was not equilibrated at all at this length scale, in sharp contrast to the fully equilibrated simulation as was done in the present paper. In other words, at the length scale of 10 lattice spacings, the chiralities are little thermalized and are virtually frozen in the non-equilibrium pattern, while only the SG correlation grows modestly in such a nonequilibrium chiral environment. After all, however, we have to understand the spin dynamics at long enough length scales at which the $Z_{2}$ chiral degree of freedom is also fully thermalized. Thus, the spin dynamics as observed in the off-equilibrium situations of Refs. 19 and 46 may not faithfully represent the close-to-equilibrium critical dynamics of the original model.

A similar dynamical simulation on the $3 \mathrm{D} \pm J$ Heisenberg SG was performed by Matsumoto, Hukushima and Takayama. ${ }^{44}$ They also made a dynamical scaling analysis taking the effect of global spin rotations into account. In this study, the time scale toward equilibrium was controlled via the analysis of the waiting-time dependence of the results. In contrast to Refs. 19 and 46, Matsumoto et al suggested that their data were consistent with the separate spin and chiral transition, i.e., $T_{\mathrm{SG}}<T_{\mathrm{CG}}$.

Berthier and Young also argued that their observation that the dynamical CG correlation length stayed shorter than the dynamical SG correlation length presented an evidence that the spin, rather than the chirality, was the order parameter of the transition. 46 Some caution is required in drawing the final conclusion from this observation, though. Both the spin and the chirality length scales probed by the off-equilibrium simulations of Refs 19 and 46 are still shorter than the crossover length estimated in the present paper $L_{\times} \simeq 20$. Hence, within the spin-chirality coupling/decoupling scenario, there still exists a good possibility that the dynamical CG correlation length eventually outgrows the dynamical SG correlation length at longer times.

In Ref. 46, the aging phenomena were persistently observed at lower temperatures, not only for the chirality but also for the spin, which was interpreted as an evidence of the occurrence of simultaneous spin and chiral transition ${ }^{46}$ Again, this cannot be taken as an unambiguous indicator of a finite-temperature SG transition, since the aging phenomena could arise simply when the time scale of measurements becomes comparable to the longest relaxation time in the system which could be extremely long in SG even in the paramagnetic phase. For example, in the 2D Ising SG which is known to exhibit no finite-temperature SG transition, clear aging phenomena have been observed both in numerical simulations 53.54 and in experiments 55.56 .

\section{Correlation length}

In Ref. 20, Lee and Young calculated by means of equilibrium simulations both the $\mathrm{SG}$ and the $\mathrm{CG}$ correlation lengths of the 3D Heisenberg SG with the Gaussian coupling in the range of sizes $4 \leq L \leq 12$. Lee and Young observed a crossing of the dimensionless correlation lengths $\xi / L$ for different $L$ for both cases of the spin and the chirality, and concluded that the spin and the chirality ordered simultaneously at a finite temperature $T / J \simeq 0.15$. The behavior of $\xi / L$ observed in Ref. 20 turned out to be quite different from that of some other dimensionless quantities, e.g., the Binder ratio, whereas Lee and Young argued that the correlation length might be the most trustable quantity to look at. Generally speaking, however, $\xi / L$ is also subject to significant finite-size effects, sometimes no better than other quantities: 37

We note that the numerical data of Ref. 20 are basically consistent with our present data for smaller sizes $L \leq 12$ : See Fig. 10(a). As emphasized in Sec. VD of our present paper, however, the crossing behavior of the dimensionless $\mathrm{SG}$ correlation length $\xi_{\mathrm{SG}} / L$ tends to change for larger lattices $L>12$ : The crossing becomes weaker and weaker, and $\xi_{\mathrm{SG}} / L$ of $L=16$ and that of $L=20$ do not quite cross with a finite crossing angle as occurs for smaller lattices $L \leq 12$, but instead, merge almost tangentially and stay on top of each other at lower temperatures: See Fig. 10(b). In contrast, the dimensionless CG correlation length $\xi_{\mathrm{CG}} / L$ of $L=16$ and that of $L=20$ persistently exhibits a clear crossing. If such a tendency continues for larger lattices, the crossing of $\xi_{\mathrm{SG}} / L$ might no longer occur for large enough lattices, at least at the crossing temperature of $\xi_{\mathrm{CG}} / L$. We thus suspect that the crossing behavior of $\xi_{\mathrm{SG}} / L$ as reported in Ref. 20 might be a transient behavior due to the small sizes, which reflected the "coupling" behavior expected at $L<L_{\times} \approx 20$. Namely, within the spin-chirality coupling/decoupling scheme, the SG correlation exhibits the ordering behavior similar to the CG correlation at shorter length scales at which the spin is coupled to the chirality, while at longer length scales at which the spin is decoupled from the chirality, the SG correlation eventually exhibits the non-ordering behavior different from the CG correlation. Unfortunately, the largest lattice size ac- 
cessible by the present computational capability $L \simeq 20$, being only comparable to the crossover length for the spin-chirality coupling/decoupling to occur, is still not large enough to clear see this behavior. We do expect, however, that the correlation lengths for larger lattices $L>20$ would eventually exhibit a clear spin-chirality decoupling behavior.

We note that such a coupling/decoupling behavior of the SG correlation length in smaller/larger lattices was indeed observed recently in the $2 \mathrm{D}$ Heisenberg SG ${ }^{24}$ For the 2D Heisenberg SG with the Gaussian coupling, Kawamura and Yonehara calculated the dimensionless SG correlation length $\xi_{\mathrm{SG}} / L$ up to the size $L=40$, and found that $\xi_{\mathrm{SG}} / L$ for the smaller sizes $L=10,16,20$ crossed almost at a common temperature $T / J \simeq 0.022$, disguising the occurrence of a finite-temperature SG transition: See the inset of Fig.6(a) of Ref. 24, while the data for the larger sizes $L=30$ and 40 data eventually came down, no longer making a crossing at $T / J \simeq 0.022$. The asymptotic non-ordering behavior observed for $L>20$ is consistent with a zero-temperature SG transition, which has been well established in 2D,23.24 Meanwhile, the CG correlation length exceeds the SG correlation length at around $T / J \simeq 0.022$, which might naturally explain the reason why $\xi_{\mathrm{SG}} / L$ for smaller sizes $L \lesssim 20$ exhibited a crossing behavior. Anyway, this observation in $2 \mathrm{D}$ gives us a warning that one should be careful in interpreting the crossing-like behavior of $\xi / L$ observed for smaller sizes as an unambiguous evidence of a true $\mathrm{SG}$ phase transition.

\section{E. Finite-size scaling of the order parameter}

Matsubara, Shirakura, Endoh and Takahashi made a finite-size scaling analysis of the SG order parameter $q_{\mathrm{SG}}^{(2)}$ for the $3 \mathrm{D} \pm J$ Heisenberg $\mathrm{SG}$, and claimed that the quality of the scaling was much better when ones assumed a nonzero SG transition temperature $T_{\mathrm{SG}} / J=0.18$ than a zero SG transition temperature $T_{\mathrm{SG}} / J=0, \frac{39}{3}$ Their conclusion is in apparent contrast to that of our present work based on a similar scaling analysis in Sec. VC We note that the quality of the finite-size scaling is sometimes sensitive to the range of lattice sizes and the range of temperatures used in the fit.

As already noticed in Sec. $\mathrm{VC}$ this point could be particularly serious in the present model. In the spinchirality coupling/decoupling scheme, the SG correlation and the CG correlation are trivially coupled at shorter length scale $L \lesssim L_{\times} \approx 20$, so that even the SG order parameter $q_{\mathrm{SG}}^{(2)}$ would be scaled for smaller sizes with assuming a simultaneous SG and CG transition, with apparent (not true) SG pseudo-exponents $\nu_{\mathrm{SG}}^{\mathrm{eff}} \simeq \nu_{\mathrm{CG}}$ and $1+\eta_{\mathrm{SG}}^{\mathrm{eff}} \simeq\left(1+\eta_{\mathrm{CG}}\right) / 3$. Indeed, as was shown in Fig. 8 our present data, particularly those of $L \lesssim 16$, turned out to be scaled reasonably well by assuming a simultaneous SG and CG transition at $T / J=0.19$, which we interpreted as a pre-asymptotic pseudo-critical behavior real- ized in the short-scale coupling regime. Furthermore, the relation between $\eta_{\mathrm{CG}}$ and $\eta_{\mathrm{SG}}^{\mathrm{eff}}$ mentioned above roughly holds at short length scale; $\left(1+\eta_{\mathrm{CG}}\right) / 3 \sim 0.60$ versus $1+\eta_{\mathrm{SG}}^{\mathrm{eff}} \sim 0.88$ at $T / J=0.19$. At longer length scales, however, the spin is eventually decoupled from the chirality. Then, if ones continues to put $T_{\mathrm{SG}}=T_{\mathrm{CG}}$ in the fit of $q_{\mathrm{SG}}^{(2)}$, the good data collapse obtained for smaller sizes would eventually deteriorate for larger sizes. Indeed, as shown in Fig. [8] our $L=20$ data of $q_{\mathrm{SG}}^{(2)}$ showed such a deviation expected for larger sizes.

Within the spin-chirality coupling/decoupling scheme, in order to see the true asymptotic critical behavior of the SG transition occurring at $T_{\mathrm{SG}}\left(<T_{\mathrm{CG}}\right)$, one has to enter into the long-scale decoupling regime and well below the CG transition temperature, i.e., $L \geq L_{\times} \approx 20$ and $T<T_{\mathrm{CG}} \approx 0.2 J$. It should be noticed that, in their scaling fit of $q_{\mathrm{SG}}^{(2)}$, Matsubara et al included the data points for smaller sizes $L=5,7,9$ etc, which are expected to lie in the short-scale coupling regime, as well as the data points at temperatures above $T_{\mathrm{CG}}$ which might lie outside the asymptotic critical regime of the SG transition. Hence, the poor scaling reported by Matsubara et al with assuming $T_{\mathrm{SG}}=0$ might simply be due to the fact that the data points used in the fit are not in the correct asymptotic regime.

By contrast, we have observed that, if we use the data points only of larger lattices $L \gtrsim 16$ and only at temperatures below $T_{\mathrm{CG}}$, the data were scaled reasonably well even with assuming $T_{\mathrm{SG}}=0$ : See Fig. 9] Therefore, we believe that there still exists a good possibility that the SG order occurs only at $T=0$ as has widely been believed in the community, although it is also quite possible that it occurs at a low but nonzero temperature, $0<T_{\mathrm{SG}}<T_{\mathrm{CG}}$.

As discussed in some detail above, any of the recent works claiming the simultaneous spin and chiral transition in the 3D Heisenberg SG appears not conclusive. As far as the authors are aware, all of these observations are consistent with the spin-chirality coupling/decoupling scheme with the crossover length scale of 20 lattice spacings and the crossover time scale of $10^{5}-10^{6}$ MCS. Rather, we believe that some of the observations reported in the present paper give a strong numerical support that the SG transition indeed occurs at a temperature below the CG transition temperature, i.e., $T_{\mathrm{SG}}<T_{\mathrm{CG}}$.

\section{SUMMARY}

In summary, we studied the equilibrium properties of the three-dimensional isotropic Heisenberg spin glass by means of extensive MC simulations. We presented evidence of a finite-temperature CG transition without accompanying the conventional SG order through the observation of various physical quantities including the order parameters, equilibrium static and dynamic correlation functions, Binder parameters and overlap- 
distribution functions, etc. Our conclusion is in contrast to some of the recent numerical studies on the same model, which claimed the simultaneous SG and CG transition. We have pointed out that the crossover length scale and the crossover time scale associated with the spin-chirality coupling/decoupling are crucially important in properly interpreting the numerical data. Around the CG transition temperature, these length and time scales are roughly estimated to be 20 lattice spacings and $10^{5} \sim 10^{6} \mathrm{MCS}$, respectively. Below these length and time scales, the spin is trivially coupled to the chirality so that the spin-chirality decoupling, i.e., the SG disorder, is difficult to observe. This might give a natural interpretation of the discrepancy between our present result and the observation of the simultaneous SG and CG transition by some other authors.

Rather, it appears to the authors that our present data for larger $L$ are hard to understand based on the standpoint of the simultaneous spin and chiral transition. Hence, while simulations on still larger lattices with $L>20$ are required to settle the issue, our present data give some support to the spin-chirality decoupling scenario for the $3 \mathrm{D}$ isotropic Heisenberg spin glass.

\section{Acknowledgments}

The authors are thankful to H. Takayama, I. Campbell, H. Katzgraber, M. Picco, H. Yoshino for valuable discussion and comments. This work was supported by the Grants-In-Aid (No. 14084204 and No. 16540341) for Scientific Research from the Ministry of Education, Culture, Sports, Science and Technology of Japan. The numerical calculations were mainly performed on the SGI Origin 2800/384 at the Supercomputer Center, Institute for Solid State Physics (ISSP), the University at Tokyo. We would like to thank particularly the ISSP Supercomputer Center for granting us exclusive use of the computer system to perform the MC simulations presented here.
* Electronic address: hukusima@phys.c.u-tokyo.ac.jp

$\dagger$ Electronic address: kawamura@ess.sci.osaka-u.ac.jp

${ }^{1}$ For a review on spin glasses, see e.g., Spin glasses and random fields, edited by A. P. Young (World Scientific, Singapore, 1997); N. Kawashima and H. Rieger, in Frustrated Spin Systems, edited by H.-T. Diep (World Scientific, 2004 Singapore).

2 R. N. Bhatt and A. P. Young, Phys. Rev. Lett. 54, 924 (1985); R. N. Bhatt and A. P. Yuong, Phys. Rev. B 37, 5606 (1988).

3 A. T. Ogielski and I. Morgenstein, Phys. Rev. Lett. 54, 928 (1985); A. T. Ogielski, Phys. Rev. B 32, 7384 (1985).

4 J. R. Banavar and M. Cieplak, Phys. Rev. Lett. 48, 832 (1982).

5 W. L. McMillan, Phys. Rev. B. 31, 342 (1985).

6 J. A. Olive, A. P. Young and D. Sherrington, Phys. Rev. B 34, 6341 (1986).

7 F. Matsubara, T. Iyota and S. Inawashiro, Phys. Rev. Lett. 67, 1458 (1991).

${ }^{8}$ H. Yoshino and H. Takayama, Europhys. Lett. 22, 631 (1993).

9 H. Kawamura, Phys. Rev. Lett. 68, 3785 (1992).

10 H. Kawamura, Int. J. Mod. Phys. 7, 345 (1996).

11 H. Kawamura, J. Phys. Soc. Jpn. 64, 26 (1995).

12 K. Hukushima and H. Kawamura, Phys. Rev. E 61, R1008 (2000).

13 D. Imagawa and H. Kawamura, J. Phys. Soc. Jpn. 71, 127 (2002).

14 D. Imagawa and H. Kawamura, Phys. Rev. Lett. 92, 077204 (2004); Phys. Rev. B 70, 144412 (2004).

15 D. Petit, L. Fruchter and I. Campbell, Phys. Rev. Lett. 83, 5130 (1999); 88, 207206 (2002).

16 F. Matsubara, S. Endoh and T. Shirakura, J. Phys. Soc. Jpn. 69, 1927 (2000).

17 S. Endoh, F. Matsubara and T. Shrakura, J. Phys. Soc. Jpn. 70, 1543 (2001).
18 F. Matsubara, T. Shirakura and S. Endoh, Phys. Rev. B 64, 092412 (2001).

19 T. Nakamura and S. Endoh, J. Phys. Soc. Jpn. 71, 2113 (2002).

20 L. W. Lee and A. P. Young, Phys. Rev. Lett. 90, 227203 (2003).

21 F. Bert, V. Dupuis, E. Vincent, J. Hammann, and J. P. Bouchaud, Phys. Rev. Lett. 92, 167203 (2004).

22 D. Imagawa and H. Kawamura, Phys. Rev. B 67, 224412 (2003).

23 M. Schwartz and A.P. Young, Europhys. Lett. 15, 209 (1991)

24 H. Kawamura and H. Yonehara, J. Phys. A 36, 10867 (2003).

25 T. Uda, H. Yoshino and H. Kawamura, cond-mat/0503132

26 K. Hukushima and K. Nemoto, J. Phys. Soc. Jpn 65, 1604 (1996)

27 E. Marinari, in Advances in Computer Simulation, edited by J. Kertész and I. Kondor (Springer Verlag, Berlin, 1998), p50.

28 H. Kawamura and K. Hukushima, Int. J. Mod. Phys. C10, 1471 (1999).

29 E. Marinari, C. Naitza, F. Zuliani, G. Parisi, M. Picco, and F. Ritort, Phys. Rev. Lett. 82, 5175 (1999).

30 F. Guerra, Int. J. Mod. Phys. B 10, 1675 (1996).

31 H. Bokil, A. J. Bray, B. Drossel, and M. A. Moore, Phys. Rev. Lett. 82, 5174 (1999).

32 E. Marinari, C. Naitza, F. Zuliani, G. Parisi, M. Picco, and F. Ritort, Phys. Rev. Lett. 81, 1698 (1998).

33 K. Hukushima and H. Kawamura, Phys. Rev. E 62, 3360 (2000).

34 M. Picco, F. Ritort and M. Sales, Euro. Phys. B 19, 565 (2001).

35 N. Kawashima and A. P. Young, Phys. Rev. B 53, R484 (1996); M. Palassini and S. Caracciolo, Phys. Rev. Lett. 82, 5128 (1999); H. G. Ballesteros et al, Phys. Rev. B 62, 
14237 (2000).

36 H. G. Katzgraber, Phys. Rev. B 67, 180402(R) (2003).

37 See, e.g., H.G. Katzgraber and I.A. Campbell, Phys. Rev. B 69, 094413 (2004).

38 H. Kawamura and D. Imagawa, Phys. Rev. Lett. 87, 207203 (2001).

39 F. Matsubara, T. Shrakura, S. Endoh and S. Takahashi, cond-mat/0309573

40 H. Kawamura, Phys. Rev. Lett. 80, 5421 (1998).

41 E. Marinari, G. Parisi and J.J. Ruiz-Lorenzo, Phys. Rev. B 58, 14852 (1998).

42 R.N. Bhatt and A.P. Young, J. Phys. Cond. Matt. 1, 2997 (1989).

43 R. N. Bhatt and A. P. Young, Europhys. Lett. 20, 59 (1992).

44 M. Matsumoto, K. Hukushima and H. Takayama, Phys. Rev. B 66, 104404 (2002).

45 M. Picco and F. Ritort, cond-mat/0405308

${ }^{46}$ L. Berthier and A.P. Young, Phys. Rev. B 69, 184423 (2004).

47 J.M. Kosterlitz and N. Akino, Phys. Rev. Lett. 82, 4094 (1999).

48 A.C. Carter, A.J. Bray and M.A. Moore, Phys. Rev. Lett. 88, 077201 (2002).

49 A.J. Bray and M.A. Moore, Heidelberg Colloquim on Glassy Dynamics, Lecture Notes in Physics Vol. 275
(Springer-Verlag, Berlin, 1986) p.121.

50 The order-pramameter space of the Heisenberg SG is $O(3)=Z_{2} \times S O(3)$, where the $Z_{2}$ refers to the chiral degeneracy and the $S O(3)$ refers to the continuous degeneracy associated with proper spin rotations. As analyzed in detail in H. Kawamura and S.Miyashita, J. Phys. Soc. Jpn. 53, 4138 (1984), the $S O(3)$ is not a simply connected space as a topological space, which enables the existence of a topologically stable point defect, $Z_{2}$-vortex.

51 F. Krzakala and O.C. Martin, Phys. Rev. Lett. 85, 3013 (2000); J. Houdayer and O.C. Martin, Euro. Phys. Lett. 49, 794 (2000).

52 M. Palassini and A.P. Young, Phys. Rev. Lett. 85, 3017 (2000).

53 J.O. Anersson, J. Mattsson and P. Svedlindh, Phys. Rev. B 46, 8297 (1992).

54 J. Kisker, L. Santen, M. Schreckenberg and H. Rieger, Phys. Rev. B 53, 6418 (1996).

55 A.G. Schins, A.F.M. Arts, H.W. de Sijn, L. Leylekian, E. Vincent, C. Paulsen and J. Hammann, J. Appl. Phys. 69, 5237 (1991).

56 J. Mattsson, P. Granberg, P. Nordblad, L. Lundgren, R. Loloee, R. Stubi, J. Bass and J.A. Cowen, J. Magn. Magn. Mater. 104-107, 1623 (1992). 